\title{
Abstracts from the 35th Annual Scientific Meeting of the Canadian Geriatrics Society, Montreal, April 2015
}

http://dx.doi.org/10.5770/cgj.18.203

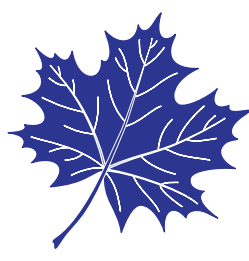

ORAL ABSTRACTS \#1-\#24

\section{A Survey of the Availability of Geriatric Expertise in Quebec Emergency Departments}

D. Cetin-Sahin ${ }^{1}$, J. McCusker ${ }^{1}$, A. Ciampi ${ }^{1}$, T.T.M. $\mathrm{Vu}^{2}, \mathrm{~S}$. Cossette ${ }^{2}, \mathrm{~N}$. Veillette ${ }^{2}, \mathrm{~A}$. Vadeboncoeur $^{2}, \mathrm{E}$. Belzile $^{3}$, F. Ducharme ${ }^{2}$. ${ }^{1}$ McGill University, Montreal, QC; ${ }^{2}$ University of Montreal, Montreal, QC; ${ }^{3}$ St. Mary's Research Centre, Montreal, QC, Canada.

Optimizing heath care services for seniors in emergency departments (ED) is a core component of the "Senior Friendly Hospital Approach" being implemented in Quebec. We measured the availability of geriatric expertise in Quebec EDs and its relationship with ED characteristics such as university affiliation, number of stretchers, and geographical location.

We surveyed (2013-2014) head nurses and head physicians at 116 adult, non-psychiatric Quebec EDs. We defined high level of availability in the three following components of geriatric expertise: 1) geriatric care coordinatioN $=$ a designated clinician coordinating the care of older adults available every day; 2) multidisciplinarity = 4 or 5 different nonmedical professionals, i.e., specialized nurse, social worker, occupational therapist, physiotherapist, and pharmacist, available almost always/often (nursereported); and 3) geriatric consultation = a specialist or a general practitioner specialized in geriatrics available almost always/often (physician-reported). We performed descriptive analyses and Fisher's exact test.

Among participating EDs $(\mathrm{N}=83), 73$ (88\%) nurses and $67(81 \%)$ physicians participated in the survey. $18 \%$ of EDs had high level of geriatric care coordination, $41 \%$ provided high level of multidisciplinarity, and 33\% received high level of geriatric consultation. We found that EDs that had greater number of stretchers were more likely to have high level of geriatric care coordination $(p<.05)$ and consultation $(p<.001)$. EDs in metropolitan areas were also more likely to receive high level of geriatric consultation $(p$ $<.01)$. High level of multidisciplinarity was not associated with any ED characteristics.

$2 / 5$ of Quebec EDs provide high level of multidisciplinarity varying in their characteristics, whereas smaller and non-metropolitan EDs lack geriatric care coordination and consultation.

There is a need for reorganisation and finding innovative ways to use existing human resources in Quebec EDs.

\section{A Pharmacist-Physician Intervention Model Using a Computerized Alert System to Reduce High-Risk Medication Use in Elderly Inpatients}

K. Arvisais ${ }^{1}$, S. Bergeron-Wolff ${ }^{1}$, C. Bouffard ${ }^{1}$, A.-S. Michaud $^{1}$, J. Bergeron ${ }^{1}$, S. Brazeau ${ }^{1}$, T. Joly-Mischlich ${ }^{1}$, N. Bernier-Filion $^{1}$, L. Lanthier ${ }^{1}$, G. Ricard ${ }^{1}$, M.-C. Rodrigue ${ }^{1}$, B. Cossette $^{1}$, L. Mallet ${ }^{2}$. ${ }^{1}$ University of Sherbrook, Sherbrook, QC; ${ }^{2}$ University of Montreal, Montreal, QC, Canada.

Background: Use of potentially inappropriate medication (PIMs) in the hospitalized elderly can lead to adverse drug events, and contribute to geriatric syndromes. PIMs are frequently prescribed to elderly hospitalized patients, indicating the need to develop strategies to reduce their use.

Objectives: To assess 1) the applicability of a pharmacistphysician intervention model to reduce the use of highrisk medications and 2) the clinical relevance of the alerts generated by a computerized alert system (CAS).

Methods: The study was conducted in patients aged 65 or older admitted to a teaching hospital between April and June 2014. In the intervention model, the pharmacist determined the clinical relevance of the CAS alerts, analyzed the patient's pharmacotherapy, and elaborated a geriatric pharmacotherapeutic plan to be discussed with the treating physician. The alerts were based on the Beers criteria. The main outcome was the change rate, defined as the number of patient-days with a change in at least one medication out of the total number of patient-days with at least one intervention.

Results: The CAS identified 200 patient-days with at least one alert. In 149 patient-days, at least one alert was 
judged to be clinically relevant for a positive predictive value of $74.5 \%$. The change rate was $77.7 \%$. The most frequent changes were drug discontinuation (40.9\%), and dose reduction (31.1\%). The most frequently targeted drug classes were bone resorption inhibitors (20.2\%), antiemetics (18.1\%), benzodiazepines (13.0\%), antidepressants (11.9\%), antipsychotics (9.8\%), and opiate agonists $(7.8 \%)$.

The inpatient geriatric consultation team was involved in $42(22.3 \%)$ of the 188 included hospitalizations.

Conclusion: The intervention model reduced high-risk medication use in hospitalized older patients, most of whom, otherwise, did not have a comprehensive geriatric assessment.

3. Does the Geriatric Anxiety Inventory (GAI) Appropriately Assess Anxiety Symptoms Among Geriatric Outpatients? A Rasch Model Approach

M. Huang, E. Lebedeva, L. Koski. McGill University, Montreal, QC.

The GAI was recently developed to address the need of a screening test that is simple, brief, and tailors to an older adult healthy and clinical population while minimizing items assessing somatic symptoms (Pachana et al., 2007). The purpose of this study is to assess the psychometric properties of the GAI as a tool to measure severity of anxiety symptoms among geriatric outpatients.

Participants $(\mathrm{N}=190)$ were recruited from two geriatric outpatient clinics at the McGill University Health Centre (MUHC). The 20-item GAI was administered in English or French. The data were analyzed via the Rasch analysis (RUMM2030) to examine unidimensionality of the construct being measured and assess individual item fit.

Unidimensionality was confirmed by good fit of all the GAI items to the Rasch model with no significant item-trait interaction. There were no residual correlations of items above 0.3 observed. The 20 -item GAI hierarchy ranged a span of 3.61 logits with the least severe anxiety symptom as "think of myself as worrier" to the most severe anxiety symptom as "feel great knot in stomach." Standard error for item estimates ranged from 0.21 to 0.43 . Person separation index was 0.71 when excluding individuals who did not report anxiety symptoms (38.4\%).

GAI items appropriately capture the anxiety construct. Even though in our sample GAI does not allow fine discrimination among individuals with similar severity of anxiety symptoms, this may be due to the development of the GAI as a screening test.

All 20 GAI items represent a unidimensional anxiety construct, hence the GAI is a valid measure to assess geriatric outpatients' anxiety symptomatology.

\section{Older Cognition Design of a Population Highly Vulnerable in Brazil}

E. Rossetti ${ }^{1}$, S.C. Pavarini ${ }^{2}$, M.S. Zazzetta ${ }^{2}$, K. Gramani ${ }^{2}$, M. Terassi ${ }^{2}$. ${ }^{1}$ University of São Carlos, São Carlos,Brazil; ${ }^{2}$ Universidade Federal de São Carlos, São Carlos,Brazil

Population aging is a global phenomenon that creates challenges in the care of the elderly population may be jeopardized in their cognitive health. The objective of this study was to delineate the cognition of registered elderly caregivers in a family-USF Health Unit in an area of high social vulnerability.

The sample consisted of 73 elderly caregivers, over the age of 60 . The interviews were conducted in the homes of elderly people in previously scheduled days and times. The instruments used for data collection were a sociodemographic questionnaire, The Addenbrooke's Cognitive Examination Revised (ACE-R), and Mini-Mental State Examination (MMSE).

Of the 73 elderly caregivers $58 \quad(73.45 \%)$ were females and $15(20.54 \%)$ were male, with a mean age of $70.35( \pm 8.50)$ years and average schooling of 2.31 years. Regarding cognitive assessment, the average total points in the ACE-R was $49.58( \pm 18.11)$ and MMSE $19.9( \pm 4.6)$. The average scores on the ACE-R were: Attention/orientation $11.84( \pm 3.15)$ points; Memory $10.45( \pm 6.05)$; Verbal fluency 4.32 ( \pm 2.84$)$; Language $14.9( \pm 5.7)$; and spatial Viso 8.02 $( \pm 3.63)$. Of the elderly, $39(53.42 \%)$ did not have changes in cognition according to the MMSE, using education by cohort note. In females $48.27 \%$ had cognitive impairment and in males $40 \%$ of the elderly.

There are few studies that bring the design of cognition of the elderly in a highly vulnerable population, showing the need to study this further.

We conclude that low educational level, the advanced age of the caregiver and the percentage of caregivers with cognitive impairment signs are worrying factors in the elderly, highly vulnerable population.

\section{Artificial Neural Network and Falls in Community- Dwellers: A New Approach to Identify the Risk of Recurrent Falling?}

A. Kabeshova ${ }^{1}$, C. P. Launay ${ }^{1}$, C. Anwweiler ${ }^{1}$, O. Beauchet ${ }^{1}$, V. Gromov ${ }^{2} .{ }^{1}$ Angers University Hospital, Angers, France; ${ }^{2}$ Oles Honchar Dnipropetrovsk National University, Dnipropetrovsk, Ukraine.

Identification of the risk of recurrent falls is complex in older adults. The aim of this study was to examine the efficiency 
of three artificial neural networks (ANNs): multilayer perceptron (MLP), modified MLP, and neuroevolution of augmenting topologies (NEAT) for the classification of recurrent fallers and non-recurrent fallers using a set of clinical characteristics corresponding to risk factors of falls measured among community-dwelling older adults.

Based on a cross-sectional design, 3,289 communitydwelling volunteers aged 65 and older were recruited. Age, gender, body mass index (BMI), number of drugs daily taken, use of psychoactive drugs, diphosphonate, calcium, vitamin D supplements, and walking aid, fear of falling, distance vision score, Timed Up\&Go (TUG) score, lower-limb proprioception, handgrip strength, depressive symptoms, cognitive disorders, and history of falls were recorded. Participants were separated into 2 groups based on the number of falls occurred over the past year: $\leq 1$ fall and $\geq 2$ falls. In addition, total population was separated into training and testing subgroups for ANNs analyses.

Among 3,289 participants, $18.9 \%(\mathrm{~N}=622)$ were recurrent fallers. NEAT using 15 clinical characteristics (i.e., use of walking aid, fear of falling, use of calcium, depression, use of vitamin D supplements, female, cognitive disorders, BMI $<21 \mathrm{~kg} / \mathrm{m}^{2}$, number of drugs daily taken $>4$, vision score $<8$, use of psychoactive drugs, lower-limb proprioception score $\leq 5$, TUG score $>9$ seconds, handgrip strength score $\leq 29(\mathrm{~N})$, and age $\geq 75$ years) showed the best efficiency for recurrent fallers identification: sensitivity $(80.42 \%)$, specificity $(92.54 \%)$, positive predictive value (84.38), negative predictive value (90.34), accuracy (88.39) and Cohen's kappa (0.74) compared to MLP and modified MLP.

Our results show that NEAT and modified MLP are both efficient ANNs for the identification of recurrent fallers, the most effective ANN being NEAT.

NEAT using a set of 15 clinical characteristics was an efficient ANN for the identification of recurrent fallers in older community-dwellers.

\section{Fear of Falling and Gait Variability in Older Adults: A Systematic Review and Meta-Analysis}

S. Ayoubi, C.P. Launay, C. Annweiler, O. Beauchet. Angers University Hospital, Angers, France.

Fear of falling (FOF) and increased gait variability are independent markers of gait instability. There is a complex interplay between them. The purposes of this study were 1) to perform a qualitative analysis of all published studies on FOF-related changes in gait variability through a systematic review, and 2) to quantitatively synthesize FOF-related changes in gait variability.

A systematic Medline literature search was conducted on May 2014 using the Medical Subject Heading (MeSH) terms "Fear" OR "fear of falling" combined with "Accidental
Falls" AND "Gait" OR "Gait Apraxia" OR "Gait Ataxia" OR "Gait disorders, Neurologic" OR "Gait assessment" OR "Functional gait assessment" AND "Self efficacy" OR "Self confidence" AND "Aged" OR "Aged, 80 and over." Systematic review and fixed-effects meta-analysis using an inverse-variance method were performed.

Of the 2184 selected studies, 10 observational studies (including 5 cross-sectional studies, 4 prospective cohort studies and one case-control study) met the selection criteria. All were of good quality. The number of participants ranged from 52 to 1,307 older communitydwellers (26.2 to $85.0 \%$ women). The meta-analysis was performed on 10 studies with a total of 999 cases and 4,502 controls. In one study, the higher limits of the effect size's confidence interval (CI) were lower than zero. In the remaining studies, the higher limits of the CI were positive. The summary random effect size of 0.29 [95\% CI: $0.13 ; 0.45]$ was significant, albeit of small magnitude, and indicated that gait variability was overall $0.29 \mathrm{SD}$ higher in FOF cases compared to controls.

This systematic review and meta-analysis shows that FOF is associated with a small significant increase in gait variability (i.e., worst performance of gait). In addition, mixed results of qualitative analysis suggest that this association may be influenced by other covariables that should be taken into account when examining it.

Our findings show that FOF is associated with a statistically significant, albeit of small magnitude, increase in gait variability.

\section{Prevalence of Behavioral and Psychological Symptoms by Stage of Dementia among Residents of Long-Term Care Facilities in Nova Scotia}

O. Veselskiy, E.G. Marshall, N. Varatharasan, M.K. Andrew. Dalhousie University, Halifax, NS.

Behavioural and psychological symptoms of dementia (BPSD) are common among older adults with dementia in long-term care (LTC) facilities. There is controversy regarding the prevalence of different manifestations of BPSD across the stages of dementia. This study examined the prevalence of BPSD in LTC in urban Nova Scotia. We also aimed to create a profile of different components of BPSD across the spectrum of dementia severity.

LTC chart reviews were conducted between September 1, 2011, and January 31st, 2012, using a Comprehensive Geriatric Assessment (CGA) tool adapted for Long-Term Care: the LTC-CGA. A total of 269 LTC residents from 10 LTC facilities (LTCF) in Halifax, Nova Scotia with documented cognitive status were included, of whom 199 had a diagnosis of dementia. Group comparison of demographic variables, cognition (assessed using the Mini- 
Mental State Examination), BPSD symptoms, and function in Activities of Daily Living (ADLs) were performed across stages of dementia severity.

BPSD were present in $76.9 \%$ of 199 residents with dementia, and a similar behavior pattern without physical aggression was also reported in up to $45 \%$ without a dementia diagnosis. The prevalence of psychological symptoms increased only slightly with dementia severity $(28 \%, 31 \%$, and $32 \%$ for mild, moderate, and severe, respectively), and were in fact most prevalent among those without dementia $(36 \% ; p<.001)$. Behavioural symptoms did increase with severity of cognitive impairment $(p<.001)$.

BPSD are highly prevalent among residents of LTC facilities in Nova Scotia.

In contrast to often-held views that BPSD are a manifestation of severe dementia, here BPSD appeared to be prevalent across the spectrum of dementia severity, and many behavioral and psychological clusters were indeed identified in residents without a documented diagnosis of dementia.

\section{Predictors of Outcomes in Slow-Stream Rehabilitation}

G. Leung ${ }^{1}$, P. R. Katz ${ }^{1}$, M. A. Binns ${ }^{1}$, G. Naglie ${ }^{1}$, J. Karuza ${ }^{2}$, A. $\mathrm{Chan}^{3}$, A. Berall ${ }^{3}$, S. Fallah ${ }^{3}$. ${ }^{1}$ University of Toronto, Toronto, ON; ${ }^{2}$ Buffalo State University, Buffalo, NY; ${ }^{3}$ Baycrest Health Sciences, Toronto, ON.

To identify the predictors of outcomes of elderly patients admitted to a slow-stream, low-intensity, long-duration inpatient rehabilitation (SSR) program after an acute hospitalization because they were unable to tolerate traditional inpatient rehabilitation.

Prospective cohort study with assessments conducted on admission and discharge. 104 patients above age 60 admitted between September 2011 and December 2012 to Baycrest's 30-bed SSR Unit in Toronto, Ontario. Admission assessments included motor Functional Independence Measure (FIM), Montreal Cognitive Assessment (MoCA), grip strength, ability to ambulate, Berg Balance Scale, Older American Resources and Services Activities of Daily Living (OARS ADL) based on functional status prior to the hospitalization, Short Form Health Survey (SF-12), Confusion Assessment Method (CAM), Patient Health Questionnaire (PHQ-9), and Comprehensive Geriatric Assessment Frailty Index (CGA-FI). Discharge outcomes included change in motor FIM from admission to discharge, residential status at discharge, and length of stay. Univariate and multivariate analyses were performed.

Mean age was $81.6 \pm 8.4$ years and $68.3 \%$ were females. Mean length of SSR stay was $82.5 \pm 26.4$ days. Mean change in motor FIM from admission to discharge was $21.0 \pm 12.2$ $(p<.001)$. Higher MoCA, higher Berg, lower CGA-FI and lower motor FIM at admission showed significant association with greater improvements in motor FIM. Lower CGA-FI was associated with returning to previous living situation. No significant predictors of lengths of stay were found in the multivariable analyses.

Lower frailty scores, higher cognitive function, lower falls risk, and lower functional independence scores at admission were associated with greater improvements in motor FIM. Lower frailty scores on admission was associated with greater likelihood of returning to previous living situation.

Admission frailty scores were strong predictors of outcomes for patients admitted to SSR.

\section{9. \#GeriMedJC: An Analysis of the Growth and Impact of the Twitter-Based Complement to the Traditional Geriatric Medicine Journal Club}

A. Gardhouse ${ }^{1}$, C. L. Wong ${ }^{1}$, L. Budd ${ }^{1}$, C. Yang ${ }^{2} .{ }^{1}$ University of Toronto, Toronto, ON; ${ }^{2}$ University of Waterloo, Waterloo, ON.

Twitter is a microblogging platform that overcomes physical barriers, allows unrestricted participation, and enables interactive discussions. Twitter-based journal clubs have demonstrated growth, sustainability, and worldwide communication, using a hashtag(\#) to trend journal club participation. To date, there is no reported Twitter-based geriatric medicine journal club. We describe the first five months of \#GeriMedJC.

(a) GeriMedJC moderates \#GeriMedJC, a monthly 26-hour asynchronous journal club that complements the live, 1-hour, traditional-format geriatric medicine journal club based at the University of Toronto. Growth metrics including number of tweets, number of participants, tweet amplification, and impressions were obtained from Symplur. Thematic analysis of tweets was performed to categorize content into areas of clinical practice, medical education, health policy, and critical appraisal.

In the first five months, @ GeriMedJC has grown 217 followers, including $30 \%, 25 \%$, and $16 \%$ from the U.K., Canada, and U.S.A., respectively. Most followers were physicians (35\%), with two-thirds representing geriatricians. There was an increase in all growth metrics for the first few months with a mean of 83 tweets, 36 retweets, 16 participants, and 34,750 impressions per journal club session. The content of tweets were most relevant to clinical practice, medical education, critical appraisal, and health policy in $39 \%, 18 \%, 16 \%$ and $4 \%$ of tweets, respectively.

There has been a steady increase in growth metrics for \#GeriMedJC with demonstrated discussion in areas of clinical practice, medical education, critical appraisal, and health policy. \#GeriMedJC is another example of using Twitter to engage international and interprofessional appraisal of medical literature. 
Future directions include demonstrating sustainability and analyzing factors associated with the growth of \#GeriMedJC.

\section{Is There Utility for Urodynamic Studies in Frail Older Persons? A Scoping Study}

M. Spencer ${ }^{1}$, A. Wagg ${ }^{2} .{ }^{1}$ University of British Columbia, Vancouver, BC; ${ }^{2}$ University of Alberta, Edmonton, AB.

Urodynamics (UD) refers to a broad range of investigations of lower urinary tract function such as bladder diaries and post-void residual measurement as well as invasive procedures such as multi-channel cystometry. According to the 5th International Consultation on Incontinence, the level of evidence supporting UD in the in frail older persons is weak.

To assess the body of evidence, a scoping study was conducted by searching OVID Medline, OVID Embase, Cochrane Central Register of Controlled Trials, CINAHL, and Web of Science. The search was limited to English studies of patients $>18$ years old, published from 1965 to November 18, 2014.

A total of 10,246 abstracts meeting the search criteria were found; studies included bladder diaries, the pad test, post-void residuals, uroflowmetry, filling and voiding cystometry, and abdominal leak point pressure measurement.

Studies involving the frail elderly were few but some themes emerged: non-invasive UD (bladder diaries and pad tests) were found useful prior to treatment and to assess treatment response. Post-void residual measurement and uroflowmetry showed benefit in preventing urinary retention when performed prior to initiation of pharmacological therapy or invasive urological procedures. Voiding cystometry was of benefit to both male and female older adults prior to surgery and in the assessment of postsurgical incontinence. UD was well-tolerated by the geriatric population.

Based on current evidence, both non-invasive and invasive UD can be considered in selected elderly patients to guide treatment decisions, especially when considering surgical intervention. More research is needed in deciding which elderly patients will benefit from UD investigation.

11. Screening for Older Emergency Department Inpatients at Risk of Prolonged Hospital Stay: The Brief Geriatric Assessment Tool

O. Beauchet, C. Launay, A. Kabeshova, L. de Decker, C. Anweiler. Angers University Hospital, Angers, France.
The aims of this study were 1) to confirm that combinations of brief geriatric assessment (BGA) items were significant risk factors for prolonged LHS among geriatric patients hospitalized in acute-care medical units after their admission to the emergency department (ED); and 2) to determine whether these combinations of BGA items could be used as a prognostic tool of prolonged LHS.

Based on a prospective observational cohort design, 1,254 inpatients (mean age \pm standard deviation, $84.9 \pm$ 5.9 years; $59.3 \%$ female) recruited upon their admission to ED and discharged in acute-care medical units of Angers University Hospital, France, were selected in this study. At baseline assessment, a BGA was performed and included the following 6 items: age $>85$ years, male gender, polypharmacy (i.e., $\geq 5$ drugs per day), use of home-help services, history of falls in previous 6 months and temporal disorientation (i.e., inability to give the month and/or year). The LHS in acute care medical units was prospectively calculated in number of days using the hospital registry.

Area under receiver operating characteristic (ROC) curves of prolonged LHS of different combinations of BGA items ranged from .50 to .57. Cox regression models revealed that combinations defining a high risk of prolonged LHS, identified from ROC curves, were significant risk factors for prolonged LHS (hazard ratio $>1.16$ with $p>.010$ ). Kaplan-Meier distributions of discharge showed that inpatients classified in high-risk group of prolonged LHS were discharged later than those in low-risk group $(p<.003)$.

Prognostic value for prolonged LHS of all combinations was poor with sensitivity under $77 \%$, a high variation of specificity (from 26.6 to 97.4) and a low likelihood ratio of positive test under 5.6.

Our results show that combinations of BGA items were significant risk factors for prolonged LHS in geriatric patients admitted to ED and discharged to acute-care medical units. However, the prognostic value for prolonged LHS of these combinations of BGA items was poor, whatever the BGA items or their combinations used.

Combinations of 6-item BGA tool were significant risk factors for prolonged LHS but their prognostic value was poor in the studied sample of older inpatients.

12. Comparison of FRAX, Garvan and Qfracture in Determining Whether Or Not to Treat in Women Attending Osteoporosis Clinics

J. Thain ${ }^{1}$, D. Aw ${ }^{1}$, L. Marshall ${ }^{1}$, O. Sahota ${ }^{1}$, N. Weerasuriya ${ }^{1}$, F. Kearney ${ }^{1}$, A. Ali ${ }^{1}$, T. Masud ${ }^{1}$, W. M. Chua ${ }^{2} .{ }^{1}$ Nottingham University Hospitals NHS Trust, Nottingham, UK; ${ }^{2}$ Royal Derby Hospital, Derby, UK. 
Fragility fracture risk assessment tools are increasingly being used to inform treatment decisions. Three commonly used tools are FRAX, Garvan, and Qfracture. However, these tools produce different results for 10 -year risks of sustaining major osteoporotic fractures and hip fractures and potentially may lead to different treatment decisions. The aim of this study was to evaluate if employing these different tools with their associated guidelines would impact on the decision to treat or not in a group of postmenopausal women.

Clinicians used a questionnaire to collect information to populate the 3 tools in patients attending osteoporosis clinics and the 10-year fracture risks were calculated. For FRAX (UK), the National Osteoporosis Guideline Group (NOGG) age-related treatment thresholds were used, and for Garvan and Qfracture, a set 20\% threshold was used to determine the need for intervention.

90 women (mean age $69.5 \pm 12.9$ years) were studied. FRAX recommended treatment in $45.6 \%(95 \% \mathrm{CI}=35.3-$ $55.9 \% ; \mathrm{N}=41)$ versus $71.7 \%(95 \% \mathrm{CI}=61.7-80.5 \% ; \mathrm{N}=$ $64)$ in Garvan and 40\% (95\% CI $=29.9-50.1 \% ; \mathrm{N}=36)$ in Qfracture. There was substantial discordance in treatment recommendation between the groups with only $17.8 \%(\mathrm{~N}$ $=16$ ) of patients having the recommendation to treat by all three tools.

Compared to FRAX-NOGG (UK), employing the Garvan tool (20\% threshold) significantly over-treated and the Qfracture tool (20\% threshold) tended to undertreat and there was substantial misclassification on whether or not to treat between the three groups.

Clinicians should be aware that choosing one fracture risk assessment tool over another (with associated treatment guidelines) may strongly influence their treatment decisions.

\section{Standard Laboratory Tests to Identify Older Adults at Increased Risk of Dementia}

S. Searle, S. Howlett, K. Rockwood. Dalhousie University, Halifax, NS.

Understanding how and why dementia occurs is key to understanding its prevention and management. Recently, standard laboratory values have been used together as an index to identify adverse outcomes in the presence of frailty. We sought to investigate whether this laboratory frailty index (the FI-Lab) was associated with a diagnosis of dementia.

A secondary analysis was done of a prospective cohort study consisting of community and institutionally dwelling individuals aged 65 and older. The FI-Lab was created from laboratory tests used in the first clinical examination of the Canadian Study of Health and Ageing. Univariate and multivariate logistic regression for a diagnosis of dementia at five years was performed to identify any association with the FI-Lab.

Of 1,013 patients, 467 were alive at follow-up, of whom 391 (83.7\%) had a follow-up diagnostic evaluation. Those 76 missing diagnosis had similar demographics to the study group. In univariate analysis, the FI-Lab (mean 0.25 ) was significantly associated with a diagnosis of dementia $(p=.003)$. In multivariate analysis the FI-Lab remained significant $(p=.019)$. Combining the FI-Clinical and FI-Lab the FI-Combined $(p<.001)$, education $(p=$ $.002)$, age $(p=.019)$ and sex $(p=.032)$ were all predictive of dementia. Sex was not significantly associated with a dementia diagnosis.

A standard laboratory index (FI-Lab), modelled after the frailty index, was associated with a future diagnosis of dementia. The FI-Lab did not include any classical dementia biomarkers. This contributes further to the possibility that dementia most often occurs in those who are frail. This further supports the potential mechanism to dementia being an impaired neurocognitive repair process.

Standard laboratory tests, when combined, are able to identify individuals who are at increased risk of dementia.

\section{Assessing Delirium Rates and Treatment Practices in a Clinical Teaching Unit}

M. von Maltzahn, S. Dumanski, M. Lenartowicz, S. A. Stewart, J. Basran. University of Saskatchewan, Saskatoon, SK.

Among hospitalized older adults, delirium is a common complication of acute illness and contributes significantly to patient morbidity and mortality. The aim of our study was to investigate the incidence of delirium among older adults admitted to the Clinical Teaching Unit (CTU) in the Royal University Hospital in Saskatoon, the factors associated with development of delirium, and subsequent management practices. This information will provide us with an opportunity to develop delirium prevention, diagnosis, and management strategies.

A chart review of 170 patients over the age of 70 at admission to the CTU was completed. A validated chart review tool was used to diagnose delirium from patient charts. Delirium diagnosis was defined as a recorded diagnosis of delirium or a documentation of "mental status change" or "acute/fluctuating" along with at least one other key term documentation (acute onset, agitation, disorganized thinking, disorientation, hallucinations, inappropriate behaviour, inattention).

The delirium rate in the sample was $30.8 \%$, $(95 \%$ CI: $[21.9 \%, 39.7 \%])$. Delirium management ranged from non-pharmacologic interventions such as reorientation and reassurance, to invasive strategies such as physical or chemical restraint (predominantly haloperidol). 
Management techniques were applied inconsistently when delirium was present.

This project has demonstrated a significant presence of delirium within the CTU, with variable management strategies. Although delirium was commonly encountered, physician documentation of delirium within the chart was rare. Furthermore, benzodiazepines were occasionally used as a first-line management tool, in contrast to the Choosing Wisely Canada guidelines.

Management of delirium requires appropriate recognition and coordinated strategies for appropriate intervention. Recognizing the significant burden of delirium on the health-care system bears important implications for health resource planning and improved patient outcomes, particularly among vulnerable elderly populations.

\section{Vaccine Mismatch and the Need for Frailty-Informed Vaccine Development}

S. Rawn ${ }^{1}$, J. McElhaney ${ }^{2}$, S. McNeil ${ }^{3}$, M. Andrew ${ }^{3}$. ${ }^{1}$ Northern Ontario School of Medicine, Sudbury, ON; ${ }^{2}$ Advanced Medical Research Institute of Canada, Sudbury, ON; ${ }^{3}$ Dalhousie University, Halifax, NS.

Influenza results in over 200,000 hospitalizations and 36,000 deaths per year in the United States. Ninety percent of influenza-related deaths occur in those older than 65. In order to appropriately advise the public and direct future vaccination strategies and research, the Canadian Immunization Research Network's Serious Outcomes Surveillance (SOS) Network calculates vaccine effectiveness by monitoring the annual burden of influenza illness resulting in hospitalization.

Surveillance for influenza is conducted at 15 SOS Network sites across Canada. All patients aged 16 years or older admitted with possible influenza (e.g., acute respiratory illness, fever) received a nasopharyngeal swab testing for influenza via reverse-transcriptase PCR or viral culture. Viral strain, vaccination status, intensive care admission, and death were recorded and compared among confirmed cases and test-negative controls.

Interim data for the 2014/15 influenza season indicate:

1. A poor match between the circulating virus and vaccine strain, with poor vaccine effectiveness in all age groups (e.g. $-25.4 \%(90 \%$ CI: $-65,4.6)$ for those older than 65);

2. $69 \%$ of hospitalized cases are older than 75 years;

3. $11 \%$ required intensive care; $8 \%$ associated mortality.

One of the defining features of aging is declining immune function. Age-related changes in T-cell function result in lessened ability of elderly people, especially those who are frail, to mount a protective immune response. Given the lack of vaccine effectiveness in a mismatch year and the high associated mortality in the elderly population, multiple strategies such as biomarkers for vaccine response, highdose vaccines, and vaccine adjuvants need to be explored to mitigate adverse outcomes in future influenza outbreaks.

Further research into frailty-informed assessments of vaccine responsiveness is imperative to reduce influenzarelated morbidity and mortality.

\section{Emergency Department Management of Elderly Patients Presenting with a Fall}

A. O'Connor, M. Dasgupta, L.-A. Fraser. Western University, London, ON.

A thorough falls assessment can successfully reduce future events and injuries in elderly patients presenting to hospital with a fall. However, the frequency with which patients receive such an assessment is not clear. We sought to characterize the management of elderly patients presenting to the ED with a fall.

Records from a single large tertiary care center ED between 2003 and 2014 were searched. A random sample of 96 charts, describing visits for a "fall" in individuals $\geq 65$ years, were selected. Charts were reviewed using a detailed pre-specified data abstraction form documenting fall management.

Of the 96 patients, mean age was 78.2 years $(\mathrm{SD} \pm$ 8.53 ), and $61.5 \%$ were women. Most (42.8\%) lived at home with family, $11.5 \%$ lived alone, and $16.6 \%$ lived in nursing homes or assisted living. Fall-related injuries were common with $54.2 \%$ sustaining a new fracture. In terms of falls assessment, only $2 \%$ patient charts had documentation of the presumed etiology of their fall. Orthostatic vital signs were performed in $1 \%$ of patients, $1 \%$ had a visual assessment, $3 \%$ underwent assessment of cognition, and $3 \%$ had a medication review. Following assessment, $69.7 \%$ of patients were discharged from the ED and $25 \%$ of patients were admitted to home hospital (4.1\% transferred elsewhere, $1 \%$ died in ED). Of those discharged, $29 \%$ of patients did not have follow-up arranged, 28\% were referred for community supports, and $14 \%$ were discharged with a new walking device. Two $(2.2 \%)$ were started on Vitamin D and calcium, and $1 \%$ on a bisphosphonate.

Very few elderly patients presenting to the ED with a fall received a proper falls assessment or treatment plan.

These results suggest a significant care gap and highlight an area of opportunity for future quality improvement. 


\section{Frailty Predicts Degree of Caregiver Burden in Older Adults Attending a Geriatric Outpatient Clinic}

T. Ringer, A. Papaioannou, A. A. Hazzan, C. Kennedy, S. Karampatos, C. Patterson, B. Misiaszek, S. Marr, T. Woo. McMaster University, Hamilton, ON.

Frailty is a multicausal syndrome including weight loss, exhaustion, and lack of strength and mobility, which puts older adults at risk for adverse outcomes including falls, hospitalization, and death. High burden in caregivers of older adults is associated with physical and psychiatric morbidities. Limited research suggests a relationship between frailty in older adults and their caregivers' selfreported burden. This study analyzed the relationship between frailty in older adults attending a geriatric outpatient clinic and their caregivers' self-reported burden.

The study population consisted of 50 older adults attending an outpatient geriatric clinic in 2013 and 2014 accompanied by a caregiver. Participants' degree of frailty was evaluated using the Fried Frail Scale (FFS). Independence in activities of daily living was assessed using the Katz Index. Patient charts were reviewed for prior diagnosis of dementia. Caregivers' self-reported burden was evaluated using the Short Zarit Burden Interview (ZBI). Multivariable linear regression was performed with ZBI score as the dependent variable, and age, gender, dementia, Katz Index, and frailty status as independent variables.

Caregivers of frail (FFS $\geq 3 ; \mathrm{N}=21$ ) patients had a mean ZBI score of 19.4 ( $\mathrm{SD}=11.3)$. Caregivers of non-frail (FFS $<3 ; \mathrm{N}=29$ ) subjects had a mean ZBI score of 13.6 (SD $=8.4$ ). Frailty and prior diagnosis of dementia significantly predicted caregiver burden. Age, Katz Index, and gender did not significantly predict caregiver burden.

Caregivers of frail older adults attending a geriatric outpatient clinic reported a significantly higher level of burden than caregivers of non-frail patients. Prior diagnosis of dementia was a significant predictor of caregiver burden.

Further examination of the relationship between frailty and caregiver burden is warranted.

\section{Identifying Geriatric Learning Needs of Physicians in Physical Medicine and Rehabilitation (PMR) to Develop a Geriatric Rehabilitation Curriculum}

\section{Chau, S. Ginsburg. University of Toronto, Toronto, ON.}

Older adults with functional impairment are cared for by physiatrists in rehabilitation. With the aging population, acquiring geriatric-related competencies will be essential. Literature is limited on geriatric learning needs in physiatry but suggests that education is suboptimal. To develop a geriatric rehabilitation curriculum, a needs assessment was conducted to understand comfort level of geriatric-related competencies and resident learning needs in physiatry residents, physiatrists, and key informants (KI).

A mixed methods approach was taken. Physiatry residents at the University of Toronto were invited to participate in a questionnaire and focus group. Physiatrists in Ontario were invited to complete a questionnaire and participate in follow-up one-on-one interviews. KIs were purposively selected to participate in both surveys. Questionnaires assessed comfort level (0 very uncomfortable, 5 very comfortable) on geriatric-related competencies. Focus groups and interviews explored learning experiences and needs in geriatrics rehabilitation. Descriptive statistics were used to analyze questionnaires. NVivo software was used for qualitative analysis within a grounded theory approach.

Eighteen (87\%) residents and 27 (21\%) physiatrists completed the questionnaire. More than $50 \%$ of residents were somewhat to very uncomfortable on geriatric topics such as age-related pharmacokinetics and dynamics, pain management, frailty, physiology of aging, and depression.

Physiatrists identified similar topics in addition to cognitive impairment (including delirium), medication management, and end-of-life care. Initial qualitative analysis sheds further light on these identified learning needs; in particular, how applicability can be enhanced in the post acute care setting.

A large proportion of physicians in physiatry do not feel comfortable on core geriatric-related competencies, which were similarly identified as learning needs by physicians in physiatry.

Initial insights may provide educators with an understanding on how to improve geriatric education in physiatry.

\section{Health Transitions Among People Aging with HIV and a General Population Cohort}

T. Brothers ${ }^{1}$, S. Kirkland ${ }^{1}$, O. Theou ${ }^{1}$, K. Rockwood ${ }^{1}$, S. Zona ${ }^{2}$, A. Malagoli ${ }^{2}$, C. Stentarelli ${ }^{2}$, C. Mussini ${ }^{2}$, G. Guaraldi $^{2}$, J. Falutz ${ }^{3}$. ${ }^{1}$ Dalhousie University, Halifax, NS; ${ }^{2}$ University of Modena and Reggio Emilia, Modena, Italy; ${ }^{3}$ McGill University, Montreal, QC.

Biological aging is a stochastic process that can be characterized by the number of health deficits individuals accumulate (i.e., their frailty index). As people with HIV exhibit excess risk for multiple age-related health problems, they might age differently than people without HIV.

Secondary analysis of four-year follow-up data from the Italian Modena HIV Metabolic Clinic cohort (MHMC; $\mathrm{N}=963$; mean age $46.8 \pm 7.1$ years; $29 \%$ women) and the Italian sample of the general community-based Survey of 
Health, Ageing and Retirement in Europe (SHARE; N = 1,391 ; mean age $65.7 \pm 8.8$ years; $56 \%$ women). Health state was quantified as the number of deficits accumulated out of 31 health variables, selected separately in each cohort. Multistate transition modelling provided probabilities of health state changes, including death.

In both cohorts, mean deficit count at four years followup showed a similar, linear relationship with deficit count at baseline; MHMC consistently had smaller standard deviations in deficit counts at follow-up. Mortality was $3.0 \%$ in MHMC and $7.7 \%$ in SHARE. Adjusted for baseline deficit count, age, and gender, MHMC participants had more deficits at follow-up than SHARE participants (RR $1.28,95 \%$ CI $1.22-1.35$ ), but mortality differences were non-significant (OR 0.85, 0.45-1.61). Adjusted mean deficits at follow-up were 8.25 (7.99-8.52) in MHMC and $6.87(6.66-7.07)$ in SHARE $(p<.001)$.

Despite differences between cohorts (e.g., HIV clinic vs. community-based, age and gender distributions) and in the health variables making up the frailty indices, HIV-positive MHMC participants and general population SHARE participants demonstrated similar patterns of deficit accumulation. MHMC participants generally accumulated more deficits and exhibited greater homogeneity in outcomes.

People with HIV appear to age in patterns similar to the general population, but experience poorer and less variable outcomes over four years.

\section{Anticholinergic Drug Burden in Persons with Dementia: The Effect of Multiple Physicians}

C. Reppas-Rindlisbacher ${ }^{1}$, K. Fung ${ }^{1}$, H. Fischer ${ }^{2}$, P. Austin ${ }^{2}$, P. Rochon ${ }^{2}$, S. Gill ${ }^{3}$, D. Seitz ${ }^{3}$, C. Tannenbaum ${ }^{4}$. ${ }^{1}$ Women's College Research Institute, Toronto, ON; ${ }^{2}$ University of Toronto, Toronto, ON; ${ }^{3}$ Queen's University, Kingston, ON; ${ }^{4}$ Université de Montréal, Montreal, QC.

Anticholinergic drug therapy may worsen cognitive performance and is particularly concerning when prescribed to older adults with dementia. Care provided by multiple physicians may contribute to this inappropriate prescribing. We explored the association between the number of unique physicians providing care and anticholinergic drug burden among older persons newly initiated on cholinesterase inhibitor therapy for the management of dementia.

A population-based cross-sectional study of community and long-term care dwelling older adults with dementia in Ontario. The number of unique physicians providing care and anticholinergic drug burden using the Anticholinergic Risk Scale (ARS) were measured within the prior year.
We identified 79,067 community-dwelling older adults and 12,113 LTC residents newly dispensed cholinesterase inhibitors. In the community (mean age 81.0 years, $60.8 \%$ women), individuals saw an average of 8 unique physicians in the prior year. The odds of high anticholinergic drug burden (ARS score of $\geq 2$ ) increased by $24 \%$ for every 5 additional physicians providing care (adjusted odds ratio, 1.24; 95\% confidence interval, 1.21-1.26). Female sex, low-income status, previous hospitalization, and higher comorbidity score were also associated with high anticholinergic drug burden. In LTC (mean age 84.3 years, $67.2 \%$ women) individuals saw an average of 10 unique physicians in the prior year. Relative to the community setting, the anticholinergic drug burden based on the number of physicians providing care was similar but attenuated in LTC.

Among older adults with dementia newly dispensed cholinesterase inhibitor drug therapy, a greater number of physicians providing care was associated with higher anticholinergic drug burden scores.

Improved communication amongst physicians, and a medication review prior to prescribing a new drug therapy, as suggested by Choosing Wisely, are important strategies to improve prescribing quality.

\section{Frailty Affects Time to Recovery of Mobility in Acutely Ill Older Adults Admitted to Hospital}

O. Hatheway, A. Mitnitski, K. Rockwood. Dalhousie University, Halifax, NS.

Older adults who become ill often demonstrate impairments in mobility and balance. Such impairments are more common in frail adults, and independently are associated with death. Even so, little work has related frailty to recovery time, which was our objective here.

This is a secondary analysis of a cohort study of 409 older adult inpatients at a Canadian teaching hospital (mean age $=81 \pm 7$ standard deviation, $64 \%$ women). Frailty was measured using a frailty index based on a comprehensive geriatric assessment (FI-CGA), at baseline (two weeks prior to admission; mean $0.31 \pm 0.10$ ), and on admission (mean $0.40 \pm 0.10$ ). Mobility and balance was quantified using the Hierarchic Assessment of Balance and Mobility (HABAM). Recovery was measured as the difference in HABAM scores between discharge and admission.

The odds of no or incomplete recovery increased by 1.06 (95\% confidence interval: $1.01-1.11)$ for each 0.1 increment in the baseline FI-CGA. Recovery was similarly dependent on age, but independent of mobility scores on admission. Frailer patients showed longer recovery times, especially with an increase in FI-CGA scores between baseline and admission $(r=0.35, p<.001)$. Recovery time was independent of age. 
Recovery has been shown to be dependent both on severity of illness and baseline level of frailty. Tracking mobility and balance can help providers, patients, and families understand the course of acute illness in older adults.

Frailer patients are at a greater risk of incomplete recovery from impaired mobility and balance. Further work into how frailty and illness severity together impact recovery - perhaps through a measure that combines the two - is needed.

\section{Atypical Antipsychotics and Weight Change in Geriatric Inpatients}

E. Yeung ${ }^{1}$, S. Chun ${ }^{2}$, T. Lau ${ }^{3}$, A. Douglass ${ }^{3} .{ }^{1}$ University of Birmingham, Birmingham, UK; ${ }^{2}$ University of Ottawa, Ottawa, ON; ${ }^{3}$ Royal Ottawa Mental Health Centre, Ottawa, $\mathrm{ON}$.

Studies suggested that antipsychotic-induced weight gain is not as much of a concern in the elderly compared to the younger population. Part 1 of this study was to determine whether atypical antipsychotics induced weight change in elderly patients with various psychiatric diagnoses. Part 2 was to identify whether certain antipsychotics induced weight change in elderly patients.

In Part 1, a retrospective chart review was done on 115 geriatric inpatients. After exclusion, patients were divided into four groups: control $(\mathrm{N}=17)$, new treatment $(\mathrm{N}=18)$, long-term treatment $(\mathrm{N}=13)$, and medication switch groups $(\mathrm{N}=8)$. In Part 2, a medication review was performed on 169 geriatric inpatients. After exclusion, patients were divided into three groups: aripiprazole $(\mathrm{N}=$ $18)$, olanzapine $(\mathrm{N}=49)$, and risperidone $(\mathrm{N}=57)$. Body weights were obtained at two different time points.

No significant weight change was observed among the control $(1.5 \mathrm{~kg})$, new treatment $(0.8 \mathrm{~kg})$, long-term treatment $(-0.3 \mathrm{~kg})$, and medication switch $(1.9 \mathrm{~kg})$ groups. No significant weight change was observed between patients with and without dementia ( 0.8 and 1.1 $\mathrm{kg}$, respectively). The weight change in the aripiprazole group $(-2.0 \mathrm{~kg} ;-2.30 \%$ from baseline) was significantly different from the weight change in the olanzapine group $(0.7 \mathrm{~kg} ;+1.87 \%$ from baseline; $p<.05)$, but not from the risperidone group $(-0.4 \mathrm{~kg} ;-0.45 \%$ from baseline) Clinically significant weight gain $(>7 \%$ increase in body weight) occurred in $14.3 \%$ of the olanzapine patients, a percentage significantly higher than the $3.5 \%$ in the risperidone group.

Although antipsychotics generally caused no weight change in the elderly population, aripiprazole and olanzapine were associated with significant weight loss and weight gain, respectively.
Prospective trials are needed to confirm whether aripiprazole and olanzapine cause weight change in the elderly population.

\section{Clinical Frailty Scale Predicts Rehabilitation Outcomes on a Geriatric Rehabilitation Unit}

J. Davies ${ }^{1}$, L. Ormseth ${ }^{1}$, D. Fletcher ${ }^{1}$, I. Gutmanis ${ }^{2}$, M. J. Borrie $^{3}$, J. Linddsay ${ }^{4} .{ }^{1}$ Parkwood Institute, London, ON; ${ }^{2} \mathrm{St}$. Joseph's Healthcare London, London, ON; ${ }^{3}$ Southwestern Ontario Regional Geriatric Program, ON; ${ }^{4}$ Lawson Health Research Institute, London, ON.

Background: As no widely accepted screening tool that predicts patient rehabilitation outcomes on a Geriatric Rehabilitation Unit (GRU) exists, determination of those who will benefit from admission and how to maximize rehabilitation gains remains challenging.

Objective: Does pre-morbid frailty, as assessed with the 9-point clinical frailty scale (CFS), predict rehabilitation outcomes?

Methods: A retrospective chart review was conducted on all patients admitted to Parkwood Institute's GRU between September 2013 and May 2014. CFS scores were assigned retrospectively based on pre-morbid health and functional status data recorded in the patient's chart. Rehabilitation outcomes, including Relative Functional Gain (RFG), and Relative Functional Efficiency (RFE) were calculated.

Results: Ninety-six (41.4\%) of the 232 GRU patients were male and $44 \%$ had experienced a lower-extremity fracture. Mean age was 82.8 years (range: 59 to 96), mean CFS on admission was 4.9 (range: 1-7), mean FIM on admission was 69.9 (range: 20-109), mean RFG was 0.43 (range: -0.21 to 0.88 ), and mean RFE was 0.02 (range: -0.01 to 0.10 ). Regression modeling that included physical health (Charlson comorbidity index [CCI], BMI, use of gait aids, Braden score, MMSE, number of admission medications), emotional health (geriatric depression scale), and demographic variables (age, sex, education, presence of extended family, living alone) suggested that CFS score accounted for $19.7 \%$ of RFG variability. An additional $17.6 \%$ of the RFG variability was explained by the combination of CCI, MMSE, Braden Score, education, and lack of extended family.

Conclusion: Of all measures considered, pre-morbid CFS score was the strongest predictor of patient rehabilitation outcomes. 


\section{Pre-Clerkship Observerships to Increase Early Exposure to Geriatric Medicine}

V. YY Xu, A. Astell, M. Leung, P. You, S. Gill, M. Gibson, C. Frank. Queen's University, Kingston, ON.

The Queen's Geriatric Interest Group (QGIG) is a studentrun initiative aimed at fostering interest in geriatric medicine. Pre-clerkship observerships have been documented as valuable methods for increasing exposure and interest in a given specialty. QGIG leaders collaborated with the Division of Geriatric Medicine to arrange observerships at St. Mary's of the Lake Hospital.

Participants were paired with a preceptor for a fourhour weekend observership on an inpatient geriatric rehabilitation unit. Participants were asked to complete surveys before and after the observership, which consisted of: (1) internally-developed Likert scale questions assessing students' experiences and interest in geriatric medicine; (2) University of California Los Angeles - Geriatric Attitudes Scale (UCLA-GAS); and (3) narrative feedback.
Forty-two students participated in the program between October 2013 and May 2014. Twenty-seven participants completed the pre-observership survey (response rate 64\%), and 22 completed the postobservership survey (response rate 52\%). All participants found the process of setting up the observership easy. The majority (72.7\%) described the experience as leading to positive changes in their attitude toward geriatric medicine and $54.5 \%$ felt that it stimulated their interest in the specialty. No statistically significant change in UCLA-GAS scores was detected $(p=.35)$. All participants agreed that the program should continue, and $90 \%$ stated that they would participate again.

Despite the lack of a statistically significant difference in attitudes, the observership program was positively received by students. They enjoyed learning about the specialty, working with the preceptor and patients, the opportunity to practise clinical skills, the ease of setting up the observership and the pace of geriatric medicine.

Structured pre-clerkship observerships may be a feasible method for increasing exposure to geriatric medicine.

\section{POSTER ABSTRACTS \#25-\#92 (25 AND 65 WITHDRAWN)}

\section{Delirium in a Geriatric Assessment Unit: Incidence, Prevalence, Effects, and Validation of a Predictive Model}

P. Desmarais ${ }^{1}$, T. T. Minh ${ }^{1}$, F. Massoud 1 , Q. D. Nguyen ${ }^{1}$, R. Tahir ${ }^{2} .{ }^{1}$ Université de Montréal, Montreal, QC; ${ }^{2}$ Centre de Recherche du CHUM, Montreal, QC.

Delirium is a frequent complication among hospitalized elderly patients and is associated with poor outcomes. Many predictive models have been elaborated to identify patients at risk of developing delirium, but none of them have been validated in a geriatric assessment unit (GAU). We sought to determine the prevalence, incidence, and effects of delirium in a GAU and to validate Inouye's predictive model for delirium in this population.

We conducted a retrospective study of consecutive admissions in a GAU at Saint-Luc Hospital (Centre Hospitalier de l'Université de Montréal) between March and December 2012. Delirium risk was assessed by using Inouye's predictive model, which is based on four risk factors at admission: visual impairment, severe illness, cognitive impairment, and high blood urea nitrogen/ creatinine ratio. Delirium cases were diagnosed with the DSM-IV-TR criteria.

One hundred thirty-six patients were included. The mean age was $85.6 \pm 5.7$ years old; $64 \%$ were women. Thirty-eight patients were diagnosed with delirium at arrival (prevalence 27.9\%); 16 patients developed delirium during their hospitalization (incidence 16.7\%). New-onset delirium was associated with prolonged hospital stay (46.1 \pm 20.4 days vs. $28.2 \pm 23.7$ days, $p<.005)$ and increased mortality $(18.8 \%$ vs. $5.0 \%, p<.05)$.

Application of the predictive model did not succeed in stratifying risk of delirium within this population (low risk: RR 1.0, intermediate risk: RR 0.97, high risk: RR 1.94; chisquare test for trend: $p=.248$ ).

Delirium is common in our GAU and is associated with prolonged length of stay and increased mortality. Application of Inouye's predictive model, validated previously in an elderly population hospitalized on a medical ward, did not predict accurately the occurrence of delirium in a GAU population.

27. Can We Quickly and Thoroughly Assess Pain in Individuals with Dementia? A Correlational Study Between the PACSLAC-II, PACSLAC, and PAINAD

M. Ruest, M. Bourque, S. Laroche, K. Bergeron-Vézina, M.P. Harvey, M. Martel, C. Rioux-Perreault, Y. TousignantLaflamme, C. Apinis, D. Proulx, G. Léonard. Université de Sherbrooke, Sherbrooke, QC.

The "Pain Assessment Checklist for Seniors with Limited Ability to Communicate" (PACSLAC) is considered by many as the reference tool to assess pain in seniors who have difficulty communicating. Despite its excellent psychometric 
qualities, clinicians often report that the high number of items of the PACSLAC hampers its use in clinical setting.

(1) To evaluate the relationship between two short assessment scales (the revised version of the PASCLAC [PACSLAC-II] and the Pain in Advanced Dementia [PAINAD]) and the original version of the PACSLAC; (2) to compare the administration times of the three tools.

46 residents in long-term care (mean age $=83 \pm 10$ yrs.) with dementia and difficulty to communicate were observed during transfer or mobilization (two potentially painful procedures) by three independent evaluators. Each of them used a different assessment tool (PACSLAC, PACSLAC-II, or PAINAD; randomly assigned). Correlational analyses were used to determine the relationship between the PACSLAC and PACSLAC-II and between the PACSLAC and PAINAD; an ANOVA was used to compare the administration times for the three tools.

The PACSLAC-II and PAINAD were both moderately correlated with the PACSLAC $(r=0.63$ and $r=0.65$; all $p$ values $<.001)$. The mean administration times for the PACSLAC-II (96 $\pm 22 \mathrm{sec}$.) and PAINAD $(63 \pm 10 \mathrm{sec}$.) were significantly lower than that for the PACSLAC $(135 \pm 29$ sec.; all $p$ values $<.001$ ).

Our results suggest that the PACSLAC-II and PAINAD can both be used to quickly assess pain in individuals with dementia.

However, given the moderate association observed and the relatively small differences in administration time, we believe that the original version of the PACSLAC should be preferred when possible.

\section{How Can We Recruit Future Geriatric Specialists? A Canada-Wide Survey of Psychiatry Residents and Protocol of a Proposed Randomized Medical Education Trial}

V. Laliberte ${ }^{1}$, M. Rappaport ${ }^{2}$, S. Rej ${ }^{2}$, D. Seitz ${ }^{3}$, M. Andrew $^{3}$, M. Davidson ${ }^{4}$. ${ }^{1}$ McGill University, Montreal, QC; ${ }^{2}$ University of Toronto, Toronto, ON; ${ }^{3}$ Queen's University, Kingston, ON; ${ }^{4}$ University of Saskatchewan, Saskatoon, SK.

Despite a rapidly aging population, there remains a shortage of geriatric specialists throughout the world. The factors associated with psychiatric residents' interest in geriatric psychiatry had not been previously examined in a nationally-representative sample.

This was an online survey of 226 Canadian psychiatry residents $(24.3 \%$ response rate). The main outcome was interest in becoming a geriatric psychiatrist. Bivariate and multivariate analyses were performed to better understand what demographic, educational, and vocational variables were associated with interest in becoming a geriatric psychiatrist.
A number of respondents had an interest in becoming a geriatric psychiatrist $(29.0 \%, \mathrm{~N}=60)$, in doing a geriatric psychiatry fellowship $(20.3 \%, \mathrm{~N}=42)$, or in doing geriatric psychiatry as a part of the clinical practice $(60.0 \%, \mathrm{~N}=124)$. Demographic characteristics (e.g., age, gender, ethnicity) did not correlate with interest in geriatric psychiatry. The variables most robustly associated with interest in geriatric psychiatry were: 1) completion of geriatric psychiatry rotation(s) before 3rd year of residency (OR 5.13 [95\% CI: 1.23-21.4]); 2) comfort working with geriatric patients and their families (OR 18.6 [95\% CI: 2.09-165.3]); 3) positive experiences caring for older adults prior to medical school (OR 12.4 [95\% CI: 1.07-144.5]); and 4) the presence of annual conferences in the resident's field of interest (OR 4.50 [95\% CI: 1.12-18.2]).

Exposing medical students to clinical geriatric psychiatry rotations that increase comfort in working with older adults may help improve recruitment of geriatric specialists.

This poster will describe the protocol of a proposed randomized trial exposing medical students to geriatric psychiatry during clerkship psychiatry rotations.

\section{Deprescribing in Australian Long-Term Care Facilities: What's Important to GPs, Nurses, Pharmacists, and Residents?}

J. Turner', J. S. Bell ${ }^{1}$, S. Shakib ${ }^{2}$, S. Edwards ${ }^{3}$, M. Stanners ${ }^{4}$. ${ }^{1}$ Monash University, Melbourne, SA; ${ }^{2}$ Royal Adelaide Hospital, Adelaide, SA; ${ }^{3}$ Southern Adelaide-FleurieuKangaroo Island Medicare Local, Clovelly Park, SA; ${ }^{4}$ Torrens University, Adelaide, SA.

Polypharmacy and multimorbidity are common among older people in long-term care facilities (LTCFs). This is associated with increased hospitalizations, adverse drug events, drug interactions, and cognitive impairment. Reducing polypharmacy may reduce adverse events and improve resident quality of life. Deprescribing refers to cessation of medications after consideration of therapeutic goals, benefits and risks, and medical ethics.

The purpose of the study was to rank factors which general practitioners (GPs), nurses, pharmacists, and residents perceive are most important when deciding whether or not medications should be deprescribed.

Discipline-specific groups of GPs $(\mathrm{N}=13)$, nurses $(\mathrm{N}=$ 6), pharmacists $(\mathrm{N}=9)$ and residents/representatives $(\mathrm{N}=6)$ associated with LTCFs were conducted in South Australia. Nominal group technique was used to discuss, explore, and rank factors each discipline perceived as important when deciding whether or not to deprescribe medications.

Participants identified a wide range of factors with considerable overlap between disciplines; however, no two 
disciplines ranked factors in the same order. The highest ranked factors for each discipline were:

- GPs - evidence for deprescribing; communication with family/resident

- Nurses - GP receptivity to deprescribing; nurses ability to advocate for residents;

- Pharmacists - clinical appropriateness of therapy for individual residents; identifying a resident's goal of care; and

- Residents - residents well-being; poor continuity of nursing staff.

The results corresponded with published frameworks for GP and patient-identified factors. This study extends those frameworks to include nurses and pharmacists. Furthermore, factors were prioritized, providing guidance for practice and future research.

Multiple factors that influence deprescribing decisions in LTCFs were identified, with each discipline having different priorities. The factors important to each discipline need to be considered in the design of deprescribing interventions in this setting.

\section{What Factors are Important to Multidisciplinary Care Teams when Deprescribing in Australian Long- Term Care Facilities?}

J. Turner' ${ }^{1}$ J. S. Bell ${ }^{1}$, S. Shakib ${ }^{2}$, S. Edwards ${ }^{3}$, M. Stanners ${ }^{4}$. ${ }^{1}$ Monash University, Melbourne; ${ }^{2}$ Royal Adelaide Hospital, Adelaide; ${ }^{3}$ Southern Adelaide-Fleurieu-Kangaroo Island Medicare Local, Clovelly Park; ${ }^{4}$ Torrens University, Adelaide, Australia.

Polypharmacy and multimorbidity are common among older people in long-term care facilities (LTCFs). Polypharmacy has been associated with increased hospitalizations, adverse drug events, drug interactions and cognitive impairment. Reducing polypharmacy may reduce adverse events and improve quality of life. Deprescribing refers to cessation of medications after consideration of therapeutic goals, benefits and risks, and medical ethics.

The purpose of the study was to rank factors that metropolitan and regional multidisciplinary groups (comprising general practitioners [GPs], nurses, pharmacists, and residents' representatives) consider most important when deciding whether or not medications should be deprescribed in the LTCF setting.

Multidisciplinary groups were convened in metropolitan and regional South Australia. Using nominal group technique, the groups discussed, explored, and ranked factors they perceived important for deprescribing.
The metropolitan group ranked "adequacy of a resident's medical and medication history" as the most important factor. The regional group ranked "identifying a resident's goal of care" the most important factor. Both metropolitan and regional groups ranked the "structure of the health system" as an important factor impacting their decision to deprescribe. Both groups identified factors relating to interdisciplinary cohesiveness as important, with the metropolitan group ranking these factors more highly.

Previous research involving GPs found a perceived "lack of evidence" for deprescribing. We discovered all health-care practitioners felt this was important. Addressing the range of factors identified may improve deprescribing success in LTCFs.

Patient-centred factors were the most important overall. However, the structure of the health system had a considerable impact on the decision to deprescribe. Metropolitan and regional multidisciplinary groups prioritized different factors. This suggests that for deprescribing to be effective, local factors should be taken into account while implementing a patient-centred, multifaceted approach.

\section{Use of a Nursing Communication Tool to Reduce Hospital Transfers in Long-Term Care: A Mixed Methods Study}

S. Ballard ${ }^{1}$, M. Peretti ${ }^{1}$, O. Lungu ${ }^{1}$, F. Tabamo ${ }^{1}$, D. CetinSahin $^{1}$, L. Alfonso ${ }^{2}$, M. Wilchesky ${ }^{3}$. ${ }^{1}$ Donald Berman Maimonides Centre for Research in Aging, Montreal, QC; ${ }^{2}$ Jewish General Hospital, Montreal, QC; ${ }^{3}$ McGill University, Montreal, QC.

Avoiding unnecessary emergency room transfers (ERTs) is recognized as an opportunity to improve health outcomes for elderly residents living in long-term care facilities (LTCFs). Prior research has identified improved communication between health-care professionals as a means of lowering ERT rates, but few studies have assessed nurses' perceptions and use of communication tools following their implementation. As such, this investigation sought to: (1) evaluate nursing use of a modified communication tool implemented in a LTCF to reduce unnecessary ERTs; and (2) explore nursing attitudes towards this tool post-implementation.

This study employed a mixed-methods approach. Intensity of communication tool use was established using chart abstraction and electronic institutional records. Semi-structured interviews were conducted with registered nurses in a Montreal LTCF currently implementing the tool.

Of the 29 nurses who participated in the study $(91 \%$ response rate), 11 had used the tool in $60 \%$ or more of 
transfer cases (high-intensity users), 8 had used the tool in approximately $50 \%$ of cases (mid-intensity users), and 10 had used the tool in less than $40 \%$ of cases (low-intensity users). More experienced nurses ( $>10$ years) tended to be low/middle users, while newer nurses tended to be more frequent users. Overall, nurses found the communication tool especially helpful for surveillance purposes, but tedious to complete during time-sensitive emergencies.

Observed differences in tool use may stem from nurses' confidence in their ability to effectively manage emergency situations. Nurses with less experience may also be more likely to adhere to protocols, in general.

ERT rates are a common benchmark for LTCF quality improvement. Given the potential applicability of nursing communication tools to other facilities, understanding how users perceive and use such tools may prove useful for future program implementations.

\section{Screening for Elder Abuse in the Geriatric Long- Term Care Setting: Adapting the Elder Abuse Suspicion Index (EASI)}

S. Ballard, M. Yaffe, M. Wilchesky. McGill University, Montreal, QC.

Elder abuse (EA) is a social phenomenon with important health implications, particularly in long-term care (LTC). Residents of LTC institutions are at high risk of EA due to their physical and mental frailties, increased dependency and vulnerability, and social isolation. Despite this, studies of EA detection and prevalence have largely neglected institutionalized seniors and seniors with cognitive impairment (CI). EA screening instruments are also not suitable for these populations. As such, the objectives of this study are: (1) to identify how an existing, rapidly administered, EA screening instrument (Elder Abuse Suspicion Index) may be adapted to reflect the realities of institution-living (including residents with $\mathrm{CI}$ ); (2) to create a LTC version of this tool informed by expert panel recommendations; and (3) to pilot the use of this newly modified instrument within a sample of LTC residents.

A mixed-methods design will be employed. Recommendations for tool modification will be obtained from a carefully selected Delphi panel of twelve healthcare professionals with experience in LTC and/or EA. The revised EA screening tool will then be administered to 75 consenting residents with varying degrees of CI.

Data from the Delphi panel questionnaires will be analyzed to identify common and conflicting viewpoints. Once the tool has been modified to reflect group consensus, content validity will be determined using specificity, sensitivity, and positive and negative predictive value metrics.
In targeting the early detection of EA in a previously untested, vulnerable population, this analysis will begin to generate insight on EA prevention measures in LTC.

The rapid detection of at-risk, institutionalized seniors may lead to an institutional response at the earliest sign of suspicion, resulting in improved health outcomes for this population.

\section{Usability, Acceptability and Perceived Barriers of a Point-of-Care Jugular Venous Pressure Device to Diagnose Heart Failure in Long-Term and Primary Care}

V. Chetram, S. Hinton, G. Heckman. University of Waterloo, Waterloo, ON.

Heart Failure (HF) patients often experience a poor prognosis, evidenced by frequent hospitalizations and limited survival. Appropriate management of HF is contingent on an accurate diagnosis. Part of the physical examination for suspected/worsening HF is assessing JVP and this can be a difficult, often underutilized maneuver. The Venous 1000 is a non-invasive, commercially available device providing a JVP measure and waveform. Our aim in this study is to assess the usability, perceived barriers, and acceptability of the device in the primary and long-term care (LTC) setting by physicians and registered nursing staff.

An exploratory, ethnographic design was utilized and data were collected through four focus-group interviews. Participants were presented with a training module and had an opportunity to use the device on a test subject. Two research assistants independently coded the transcripts.

Initial results indicate a high degree of acceptability and usability, despite difficulty in locating the fourth intercostal and interpreting the JVP. Barriers include resident behavior, interprofessional communication/ structure, perceived adequacy with current clinical skills, and a belief that HF patients were appropriately diagnosed despite rarely measuring JVP.

There is an evident lack of utility of JVP despite its having a role in the Canadian Cardiovascular Society Guidelines for diagnosing Heart Failure. The device appears best suited for long-term care and utilized by nursing staff due to the nature of physician availability and lack of diagnostic investigative tests. Educational initiatives are needed for nursing staff and physicians and our findings can help direct development of future technological devices and their implementation in these settings.

This device has tremendous potential to help establish new-onset or worsening Heart Failure by providing a jugular venous pressure measurement and waveform. 


\section{Geriatric Preoperative Assessment of Elderly Patients with Gastrointestinal Cancer: Clinical Factors and Prognostic Implication}

\author{
C. Baillargeon, F. G. Idiamey. Université de Montréal, \\ Montreal, QC.
}

Patient selection for surgical oncologic treatment is a challenge, particularly with elderly patients. The purpose of this study was to compare patient's characteristics with geriatrician recommendation as fit or non-fit for surgery and to identify baseline characteristics associated with adverse immediate postoperative outcomes.

We conducted a retrospective study of patients seen in our geriatric oncology clinic before an elective surgical intervention for gastrointestinal cancer between 2010 and 2014. Patients were referred by surgeons or oncologists. Clinical and geriatric assessment variables and postoperative data were collected by chart review.

Univariate analyses were used to identify baseline patient's characteristics associated with decision prior to surgery and with postoperative outcomes (length of hospital stay and discharge status).

Forty-four patients were included (14 had hepatic metastasis of a colorectal cancer, 13 had rectal cancer, 7 had pancreatic adenocarcinoma, 3 had colon cancer, and 7 had other types). Median age was 80.1 years (70-89). Nine patients $(20.5 \%)$ were advised against surgery; they were more dependent for IADLs $(p=.003)$, had lower grip strength $(p=.003)$ and lower gait speed $(p=.029)$. Twentythree patients were operated. The median hospital stay was 10 days. Eighteen patients (78\%) had complications: 13 minors and 5 majors, including 1 death. Seven patients had delirium. Falls in the last 6 months $(p=.022)$ and polypharmacy $(p=.043)$ were associated with prolonged hospital stay. Eight patients (36\%) were discharged in rehabilitation or convalescent unit: they had lower grip strength $(p=.019)$.

Low grip strength is associated with postoperative discharge in convalescent or rehabilitation unit in our study. A prospective study is needed to confirm the results.

Low gait speed and grip strength seem to influence preoperative decisions in our geriatric oncology clinic.

\section{Verbal Repetition in Patients with Dementia: Results from an Online Survey}

P. Molin 1 , K. Rockwood ${ }^{1}$, M. Richard ${ }^{2}$. ${ }^{1}$ Dalhousie University, Halifax, NS; ${ }^{2}$ DGI Clinical Inc., Halifax, NS.

The Web allows access to people with dementia and their care partners who can provide information about important symptoms that might otherwise receive little attention.
Verbal repetition in dementia is common, troubling, and little studied. The objectives were to see how often and at which stage verbal repetition was tracked in an online list of symptoms, how it related to other symptoms, and whether frequency was less in patients on a cholinesterase inhibitor.

Verbal repetition data came from the DGI Clinical website. Care partners of people with dementia selected symptoms for tracking from a 60-item SymptomGuide.

Of 1,665 respondents, 895 reported a dementia diagnosis, in whom verbal repetition was identified as a target symptom in 267. Compared to those in whom verbal repetition was not targeted, those in whom it was were more often women $(70 \%$ vs $60 \%, p<.01)$ and more likely to have mild dementia ( $43 \%$ vs $22 \%, p<.01)$. When reported, verbal repetition was associated with symptoms of irritability, frustration, misplacing objects, and impaired recent memory. The most common descriptions were repeating the same questions about upcoming events $(98 \%)$ or the same stories at successive encounters $(94 \%)$. Verbal repetition was reported occurring 10 times/day in patients treated with a cholinesterase inhibitor vs 20/day in those not treated.

Verbal repetition can be seen at all stages of dementia, but most commonly is targeted to track treatment in the mild stages. Repetitive questioning about upcoming events was the most frequent symptom. Treatment with a cholinesterase inhibitor was associated with reports of fewer episodes of verbal repetition.

Verbal repetition is a commonly targeted symptom in the mild stages of dementia that might respond to treatment.

\section{Senior Medical Students' Experiences with Goals- of-Care Discussions: A Qualitative Study of Informal Training During the Internal Medicine Clerkship}

X. M. Wang, M. Swinton, J. J. You. McMaster University, Hamilton, ON.

Goals-of-care discussions occur between patients, family members, and clinicians to establish decisions about plans of care, including the use or non-use of life-sustaining treatments. In previous studies, learners have reported insufficient training in end-of-life communication, but most studies have focused on development or evaluation of specific educational interventions. The objective of this study was to explore senior medical students' experiences with goals-of-care discussions, specifically 1 ) whether reallife informal training reinforces or subverts formal training, 2) what behaviours are role-modelled by residents or staff physicians, and 3) whether students are observed or receive feedback on their discussions.

We conducted one-on-one, semi-structured interviews with consecutive McMaster University medical students 
who responded to our study invitation during or after their Internal Medicine clerkship. We analyzed a subset of interview transcripts in triplicate, using conventional content analysis to develop a consensus list of codes and organize them into meaningful themes. Data were analyzed concurrently with data collection to inform further interviews.

To date, we have completed coding and analysis of 5 interviews. Preliminary data suggest that students 1) believe goals of care discussions are important, 2) conduct discussions independently after observing residents, 3) feel uncomfortable conducting these discussions, 4) are not observed and do not receive feedback on their discussions, and 5) perceive significant differences between their formal and informal training.

Medical students have identified significant educational gaps around goals-of-care discussions. Despite lack of experience and supervision, they routinely conduct these discussions, raising concerns about the quality of decisions made based upon these conversations.

Our findings suggest medical students need improved education on goals-of-care discussions. This may be achieved through adoption of a competency-based curriculum.

\section{Caregiver-Delivered Delirium Prevention in Hospitalized Patients - a Pilot Study}

R. Biswas, M. Dasgupta, C. Brymer, M. Mrkobrada. Western University, London, ON.

A non-pharmacologic, family-delivered intervention directed at modifying delirium risk factors was found to prevent delirium in Chile. The feasibility of doing a similar delirium prevention intervention was tested in a pilot study at the London Health Sciences Centre (LHSC).

Eligible participants were free of delirium on admission and were community-dwelling seniors $(\geq$ 70 years of age) consecutively admitted to the medical units. Patient-caregiver dyads were randomized (stratified by age and known dementia diagnosis) to receive a delirium prevention pamphlet (with a bedside explanation of ways to prevent delirium) or a general health brochure (placebo). Caregivers were asked to complete a survey and maintain a log of the time spent visiting the patient. The Confusion Assessment Method was used for delirium diagnosis.

Out of 146 eligible patient-caregiver dyads, 79 (54.1\%) agreed to participate (67 of 146 eligible caregivers either refused or were unavailable to participate). Five patients $(6.7 \%)$ became delirious in hospital: 2 patients $(5.1 \%)$ in the placebo group and 3 patients $(7.5 \%)$ in the intervention. Surveys were returned by $52(66 \%)$ caregivers. Challenges caregivers faced were parking costs and the overall time required (average of 5.6 hours per day were spent by caregivers). Caregivers did not find the study difficult to participate in (mean score 1.9 on 1 to 5 Likert scale; in the intervention group, mean score 1.8), and 51 of $52(98.1 \%)$ of respondents found the overall experience at least somewhat rewarding.

Although $45.9 \%$ of caregivers were not interested in participating in this study, those who did participate found it to be a rewarding experience.

A family-delivered delirium prevention intervention may be another way to decrease delirium.

\section{Androgen Deprivation Therapy for Men with Prostate Cancer and the Risk of Developing Parkinsonism}

J. Young, C. Marras, R. Sutradhar, L. Yun, S. Alibhai. University of Toronto, Toronto, ON.

There are anecdotal and case reports of the development or worsening of Parkinsonian symptoms after the initiation of androgen deprivation therapy (ADT) in men with prostate cancer. Medical castration using gonadotropinreleasing hormone $(\mathrm{GnRH})$ agonists (e.g., goserelin, leuprolide) results in approximately $90 \%$ reductions in circulating testosterone, and reduction of estradiol levels by $80-90 \%$. Literature from animals and humans suggests that androgens and estrogens have important effects on the nigrostriatal dopaminergic system but their relative contributions, and how those are influenced by androgen deprivation, is uncertain.

This is a propensity-matched cohort study using linked administrative databases at the Institute for Clinical and Evaluative Sciences (ICES) and the Ontario Cancer Registry (OCR). We will compare men with prostate cancer receiving at least 6 months of ADT with those not receiving ADT. A separate non-matched cohort including all men who receive ADT will be examined to determine if the duration of ADT has an effect on the risk of developing PD.

Primary outcome: New diagnosis of Parkinson's - We will identify men who receive a new diagnosis of Parkinson's disease if they received one hospital discharge or two physician outpatient service claims with the following ICD codes: G20.X (ICD-10) or 332.0 (ICD-9).

Statistical Analysis: For the primary outcome, Cox proportional hazards models will be used based on the groups defined. Unadjusted and then adjusted hazard ratios will be calculated, along with $95 \%$ confidence intervals, using the following covariates: total duration of ADT, age, history of stroke.

Results and analysis anticipated to be available by March 2015, and ready for presentation at CGS in April. 
39. Depression Case Finding in Parkinson's Disease Patients: A Systematic Review and Meta-Analysis of Screening Tools

Z. Goodarzi ${ }^{1}$, K. Mrklas ${ }^{1}$, D. J. Roberts ${ }^{2}$, T. Pringsheim ${ }^{2}$, J. M. Holroyd-Leduc ${ }^{2}$, N. Jette ${ }^{2}$. ${ }^{1}$ Alberta Health Services, Calgary, AB; ${ }^{2}$ University of Calgary, Calgary, AB.

Background: Parkinson's disease is a neurodegenerative disorder which is very prevalent in older individuals. Depression is a common non-motor symptom of Parkinson's disease (PD), with an estimated prevalence of $35 \%$. However, the detection of depression can be challenging in this population.

Objective: To examine the diagnostic accuracy of depression screening tools for case-finding in adult outpatients with Parkinson's Disease (PD), as compared with psychiatric diagnosis based on DSM or ICD criterion.

Methods: We conducted a literature search of MEDLINE, PsychINFO, EMBASE, Cochrane Central Register of Controlled Trials, and Cochrane Database of Systematic Reviews from database inception until October 28, 2014. All citations, full text articles, quality assessments, data extraction, and analysis were independently examined by two authors. Study eligibility criteria were diagnostic accuracy studies involving adults with PD in an outpatient setting that validated a depression screening tool compared to a gold standard interview (DSM or ICD). Meta-analysis of results was completed where feasible.

Results: From a total of 7,196 citations 21 studies were included, which incorporated 15 tools. Prevalence of depression pooled from 17 unique study populations was $29.4 \%(95 \%$ CI $23.3,35.5)$. We were able to perform metaregression analysis for three screening tools. The GDS-15 (N $=6$ studies) had a pooled sensitivity of 0.85 (95\% CI 0.78 , $0.90)$ and specificity of 0.86 (95\% CI $0.81,0.89)$. The BDI-I/ Ia $(\mathrm{N}=4$ studies $)$ had a pooled sensitivity of $0.79(95 \% \mathrm{CI}$ $0.68,0.90)$ and specificity of 0.85 (95\% CI $0.79,0.96)$. The MADRS ( $\mathrm{N}=4$ studies) had a pooled sensitivity of $0.74(95 \%$ CI $0.6,0.83)$ and specificity of $0.93(95 \%$ CI $0.85,0.97)$.

Discussions: Screening tools for depression case-finding assist clinicians to identify patients who require further assessment. These tools are not diagnostic, but use of accurate tools may help facilitate appropriate use of specialty mental health services.

Conclusions: There are many validated tools available to screen for depression in PD. Based on the current evidence, the GDS-15 is an accurate tool to use for depression casefinding in PD patients, which is also favourable from a logistical standpoint given its ease of use, absence of copyright protection, and lack of overlapping questions with PD symptomatology.

\section{Should Repetition Trials, Cued and Multiple-Choice Recall Be Scored on Montreal Cognitive Assessment (MoCA)?}

E. Lebedeva, L. Koski. The Research Institute of the McGill University Health Centre, Montreal, QC.

The MoCA is widely used for Mild Cognitive Impairment (MCI) screening in elderly individuals. Memory is assessed by a free recall of 5 words, which is done after two trials of repetition. Providing category or multiple-choice cues is optional. Using Rasch analysis, we investigated whether scoring the two trials of repetition, cued and multiplechoice recall improves MoCA's psychometric properties and ability to distinguish among three groups: No Cognitive Impairment (NCI), MCI, and Alzheimer's Disease (AD).

People with NCI $(\mathrm{N}=35), \mathrm{MCI}(\mathrm{N}=197)$, and AD $(\mathrm{N}=$ 122), who were administered MoCA in the course of normal clinical care, were selected from a geriatric database. Rasch analysis was performed using two new scoring methods, which were compared to the original scoring: 1) each word for both repetition and recall was scored separately; 2) items Repeat1, Repeat2, RecallFree, RecallCue and RecallMultipleChoice each reflected performance on the set of 5 words.

Significant difference between the mean ability values of the groups was observed for original and both new scoring methods (NCI $>$ MCI $>$ Dementia, $p<.0001$, one-way ANOVA). Separation of AD and MCI was better (smaller overlap and higher difference in mean values) than separation of MCI and NCI groups. Although new scoring methods equally improved sensitivity to distinguish persons by their ability at the individual level (Person Separation Index 0.86 for both new vs. 0.84 for original scoring), they negatively affected separation of the groups.

Original and both new scoring methods allow better discrimination of AD vs. MCI than NCI vs. MCI. Observed difference among the three groups proves MoCA's discriminant validity.

Measuring and screening properties of the same tool can be affected in opposite ways while introducing changes in scoring.

41. Automatic Self-Transcending Meditation Improves Neuropsychological Symptoms in Late-Life Depression

H. Hirjee, A.M. Burhan, P. Maldeniya, M. Raza, S. Wetmore, R. Newman, A. Vasudev. University of Western Ontario, London, ON. 
Late-Life Depression (LLD) affects 3-5\% of older Canadians. Unfortunately, it is inadequately treated with antidepressants, necessitating additional treatment options. Various mind-body therapies are being embraced by patients as they are considered safe and potentially effective. Sahaj Samadhi, a form of Automatic Self-Transcending Meditation (ASTM), may offer particular benefit in LLD.

We are conducting a large single-centre, single-blind, longitudinal randomized controlled naturalistic trial to determine if ASTM improves depressed mood, anxiety, and quality of life in patients with LLD $(\mathrm{N}=96)$. Patients with LLD are randomized either to ASTM plus treatment as usual (TAU) or TAU alone. ASTM training, provided by certified teachers from the Art of Living Foundation, is administered for four consecutive days (120 minutes per day) in the first week, followed by 60-minute sessions in each of the 11 subsequent weeks. Participants are assessed at baseline (week 0), and 4, 8, and 12 weeks into the study.

With 25 patients (ASTM=11; TAU=14) having completed the 12 weeks of monitoring, we found that ASTM led to improvement in Hamilton Depression (HAM-D 17) scores from 15.5 at baseline to 8.5 at week $12(p<.001)$ with 7 of 11 treated patients achieving remission with a score below 8 . A significant ( $p=.006$ ) clinical improvement was also noted using the Clinical Global Impression (CGI) scale, and Geriatric Depression Scale (GDS) scores improved from a mean of 9.3 to 6.2 ( $p=.03$ ).

In comparison, subjects in the TAU group showed no significant differences in scores over time $(p>.05)$ other than a worsening on CGI $(p=.02)$.

Upon study completion, if ASTM continues to show effectiveness, it could be readily adapted into routine clinical care and delivered in a range of settings at relatively little cost.

\section{Appropriateness of Indwelling Urethral Catheter Insertions in the Emergency Department}

A.-Y. Ma, A. Wagg, K. Hunter, B. Rowe. University of Alberta, Edmonton, AB.

Adherence to catheter guidelines is poor and may be a particular problem in emergency departments (ED). We aimed to describe catheter insertion practice in the ED of an urban Western Canadian hospital to inform a catheter reduction strategy.

Prospective convenience sample of adult patients catheterised in the ED. Data on patient demographics, diagnosis, indications and perceptions of catheter appropriateness from chart review and interviews. Appropriateness was determined by the research team using current guidelines. Beliefs regarding catheterisation were collected by surveys.
Of 150 cases of catheterisation, $62.7 \%$ occurred in patients over $65,43.3 \%$ had a written order and $5.3 \%$ a documented reason. Based on guidelines, $58.7 \%$ were deemed inappropriate. Nurses identified more than one reason in most cases, with close monitoring of urine output for critically ill patients cited in $42.2 \%$. Of the inappropriate cases, $24.7 \%$ occurred in non-critically ill patients, $24 \%$ to manage urinary incontinence, $19.3 \%$ for mobility impairment, and $18 \%$ for confusion/dementia. Survey data revealed $96.7 \%$ of physicians and nurses perceived close monitoring of urine output for critically ill patients an appropriate indication. Both groups viewed gross haematuria (56\% and $77.2 \%$ respectively) and stroke (32\% and $55.9 \%$ respectively) as appropriate indications.

There was likely a difference in understanding of the phase "critically ill," a frequent nurse-identified reason. Of concern is the large proportion catheter insertions designed to manage urinary incontinence, mobility, or cognitive deficits. Current guideline criteria do not address gross haematuria and stroke, which nurses and physicians identified as appropriate indications.

Current practice guidelines provide only general indications for appropriate catheter use and there is a need for novel knowledge translation strategies within the ED environment to encourage appropriate catheter use.

\section{The Effect of ApoE Genotype, Plasmalogen Biosynthesis, and Reverse Cholesterol Transport Biomarkers on Cognition in Elderly Persons}

D. Goodenowe ${ }^{1}$, V. Senanayake ${ }^{1}$, T. Smith ${ }^{1}$, A. Mochizuki ${ }^{1}$, B. Chitou ${ }^{1}$, S. E. Leurgans ${ }^{2}$, D. A. Bennett ${ }^{2} .{ }^{1}$ Phenomenome Discoveries Inc., Saskatoon, SK; ${ }^{2}$ Rush University Alzheimer's Center, Chicago, IL.

ApoE genotype, serum plasmalogen (PlsEtn) levels, and biomarkers of reverse cholesterol transport (RCT) have been shown to be associated with cognition in elderly persons.

Using a community-based sample of 904 wellcharacterized elderly persons from the Rush University Religious Orders Study and Memory and Aging Project, the relationships between ApoE genotype, serum PlsEtn, triglycerides (TAG), cholesterol (CHO), $\mathrm{HDL}$ and the $\mathrm{CHO} /$ HDL ratio (HDLr) levels on cognition were measured. Effects of serum PlsEtn status was assessed using a quantitative PlsEtn Biosynthesis Value (PBV) that was generated for each person by combining the relative serum levels of three key PlsEtn species. ApoE allele effects were assessed by combining four allele combinations into one of three genotype groups $(\varepsilon 2=$ $\varepsilon 2 \varepsilon 3, \varepsilon 3=\varepsilon 3 \varepsilon 3, \varepsilon 4=\varepsilon 3 \varepsilon 4+\varepsilon 4 \varepsilon 4)$.

Using a base model that corrected for age, education, and gender we assessed the effects of each variable independently and collectively on cognition. When all 
variables were considered, only PBV (coef $=0.606, p<$ $.0001), \operatorname{ApoE}(\varepsilon 3=$ ref.); $\varepsilon 2($ coef $=0.156, p=.007) \varepsilon 4$ (coef $=-0.236, p<.0001)$, and HDLr (coef $=-0.534, p=.015)$ remained significantly associated with cognition. PBV and ApoE did not interact with each other. Both ApoE (coef = $0.173, p=.02$ ) and PBV (coef $=0.226, p=.004)$ interacted with HDLr. No effect of gender was observed.

The different ApoE alleles have different cholesterol efflux capacities with $\varepsilon 2>\varepsilon 3>\varepsilon 4$. Increasing membrane concentration of DHA-PlsEtn increases cholesterol efflux. The ratio of total cholesterol to HDL is a measure of RCT capacity. These data suggest that low RCT capacity may be a common underlying mechanism associated with lower cognition.

Metabolic and genetic mechanisms affecting RCT have a negative effect on cognition in the elderly.

\section{Supporting Family Caregivers of Seniors with Complex Needs}

L. Charles ${ }^{1}$, J. Parmar ${ }^{1}$, S. Bremault-Phillips ${ }^{1}$, J. Triscott ${ }^{1}$, P. G. $\operatorname{Tian}^{1}, \mathrm{M}$. Johnson ${ }^{2}$. ${ }^{1}$ University of Alberta, Edmonton, AB; ${ }^{2}$ Network of Excellence in Seniors' Health and Wellness, Covenant Health, Edmonton, AB.

Family caregivers are often overwhelmed by the stresses of caregiving, conflicting with other demands like work and childcare, resulting in deterioration of their health. Caregivers, thus, require support to ensure that they can continue to provide care, while maintaining their own well-being. However, there is a gap between what is known to help caregivers and what is available. This study will determine the strengths, gaps, and barriers to the provision of caregiver supports, including resource utilization and costs, and research priorities.

A CIHR-funded conference was held in Edmonton on April 14-15, 2014. On Day 1, the conference brought together over 120 researchers, health-care providers, community organizations, government, and caregivers to discuss ways to understand and support family caregivers of seniors. On Day 2, 76 participants focused on research needs for caregivers of seniors with complex needs. NVivo was utilized to support ethnographic thematic analysis.

On Day 1, barrier/gaps were identified: caregiving involves managing a multitude of tasks impacting caregivers' ability to tend to their own personal needs; changing roles and obligations; inadequate communication and information; scattered resources; need for system navigation; negative economic impact; and lack of supportive policies. On Day 2, initial research priorities were identified: defining caregivers; timing and type of support; 'Goals of Care' for caregivers; enhanced case management; system navigation supports; impactful change and funding models; and knowledge exchange strategies.
A 5-year research program was proposed with two key priorities: a longitudinal study of caregivers and their needs, and an economic cost analysis.

There's a need for centralization and navigation of resources with enhanced case management, goals of care for caregivers, education for health-care professionals and caregivers, and the development of provincial frameworks and policies.

\section{Role of Routine Tests in the Evaluation of Frailty in Acutely Ill Older Adults}

X. Wang ${ }^{1}$, K. Rockwood ${ }^{2}$. ${ }^{1}$ Jilin University, Jilin, China; ${ }^{2}$ Dalhousie Universtity, Halifax, NS.

Older people at an increased risk of adverse health outcomes compared with others their own age are said to be frail. How best to assess frailty in acutely ill older adults is disputed. In this observational cohort study, patients from the emergency department were evaluated using routine tests, and a standardized comprehensive geriatric assessment (CGA). We compared a frailty index based on a CGA (FI-CGA) with one constructed from clinical and laboratory data (FI-Lab) to determine which was better at predicting the risk of death.

Consecutive older adults (aged 65+) were enrolled and followed for at least 6 months. The FI-CGA was comprised of 55 items and the FI-Lab, which included clinical, laboratory, and ECG data, was comprised of 24 items. Kaplan-Meier plots were done to illustrate the event curves by frailty status. Predictive validity for 6-month mortality was tested by Cox proportional hazards analysis.

Of 438 participants, 109 (55 men and 54 women) died during 6-month follow-up. Mean FI scores in the least frail group were $0.151 \pm 0.033$ using the FI-CGA vs. 0.148 \pm 0.043 using the FI-Lab, and $0.653 \pm 0.048$ (FI-CGA) vs. $0.660 \pm 0.037$ (FI-Lab) in the frailest group. Mortality increased significantly as the frailty scores increased, both for FI-CGA and FI-Lab. An age- and genderadjusted model showed a clear dose-response relationship of the FI with mortality. The hazard ratios for FI-Lab and FI-CGA were 1.553 (1.334 to 1.808) and 1.380 (1.186 to 1.605), respectively. The impact on the discriminative ability of combining both FIs was modest: the area under the receiver operating characteristic (ROC) curve was 0.653 for the FI-CGA, 0.663 for the FI-Lab and 0.690 for the combined FI.

Frailty can be screened using either the standard FI (FI-CGA) or routinely collected laboratory and clinical data (FI-Lab). Both of FI-CGA and FI-Lab, singly or jointly, could identify acutely ill older patients at increased risk of death. 
Compared with the traditional method for calculating the FI (FI-CGA), the novel FI-Lab, based on routine clinical data and laboratory data, was more easily collected for clinicians and requires minimal participation by patients. Therefore the novel FI-Lab may be more suitable to measure frailty in patients with serious conditions. Further evaluation of this approach in clinical settings is encouraged.

\section{Activity Profile and Energy Expenditure in Highly Active Older Adults}

K. Madden, M. C. Ashe, J. Chase. University of British Columbia, Vancouver, BC.

Time spent in moderate-to-vigorous activity (MT) in young adults is the best predictor of daily caloric expenditure (CE); conversely $\mathrm{CE}$ is best predicted in older adults by time spent in light activity (LT). Some studies have suggested that increasing MT in older adults has no impact on CE due to a compensatory increase in daily sedentary time (ST). We examined highly active older adults to examine the biggest contributors to energy expenditure in this population.

54 community-dwelling men and women $>65$ years of age (mean 71.5 years) were enrolled in this cross-sectional observational study. All were members of the Senior's Whistler Ski Team and all met current Canadian guidelines for physical activity. Activity levels (ST, LT, and MT per day) were recorded with accelerometers worn continuously for 7 days. CE was measured using accelerometry, galvanic skin response, skin temperature, and heat flux (SenseWear armband). Significant variables were then entered into a stepwise multivariate linear model containing activity levels, age and gender.

The average proportion of time spent at a sedentary activity level each day was $72 \%$. The main predictors of higher $\mathrm{CE}$ were time spent in moderate-to-vigorous activity (MT, Standardized $\beta, 0.360 \pm 0.086, p<.001$ ) and male gender (Standardized $\beta, 1.421 \pm 0.171, p<.001$ ). A model containing only MT and gender explained 66 percent of the variation in CE. An increase in MT by one minute per day was associated with an additional 13 calories expended in physical activity.

The relationship between activity intensity and $\mathrm{CE}$ in athletic seniors is similar to that observed in young adults.

Active older adults still spend a substantial proportion of the day engaged in sedentary behaviours.

\section{Driving and Dementia: Communication Multimedia Module}

A. M. Byszewski 1 , F. Molnar ${ }^{2}$, F. Aminzadeh ${ }^{2}$, B. Power ${ }^{3}$, R. Parson ${ }^{3}$, L. Lee ${ }^{4}$. ${ }^{1}$ The Ottawa Hospital, Ottawa, ON;
${ }^{2}$ Regional Geriatric Program of Eastern Ontario, Ottawa, ON; ${ }^{3}$ University of Ottawa, Ottawa, ON; ${ }^{4}$ Memory Clinic, The Centre for Family Medicine, Kitchener, ON.

For the person with dementia (PWD), the loss of driving privilege is often worse than a diagnosis of cancer. Physicians in Canada are placed in a position where they are legally responsible and liable to report unfit drivers. Skills such as empathy, clear communication, referral to resources, and follow-up are crucial. In some cases the discussion is "rushed" within the context of multiple medical issues, leaving the PWD and caregivers with a strong emotional reaction and lack of information.

We developed a module (1.5-2 hrs) containing background material on issues specific to dementia and driving, communication strategies, links to resources, and representative video clips. Two videos were produced (demonstrating the less and more ideal), simulating reallife disclosure and management of emotionally charged discussion that often ensue. The videos were pilot tested at a CME event for Family Physicians, using an adapted form of the Calgary Cambridge Communication scale, to ensure validity. The module was then presented to postgraduate trainees at an academic half day. A pre- and post-test was administered to evaluate the effectiveness of the module.

Using the adapted Calgary Cambridge Communication Scale, validity was established regarding accurate demonstration of contrasting communication styles of the two videos. Results from pre and post survey of the module demonstrated that confidence increased $(p<.0001)$, as well as comfort and willingness in discussing the subject.

This project involved the development and evaluation of a module which demonstrated positive impact on health professionals' attitude for communication on driving cessation with PWD.

This module can to add to teaching methods around the topic of driving and dementia, such as lectures and workshops.

\section{Enoxaparin Use in a Geriatric Patient with Chronic Kidney Disease: A Report of Retroperitoneal Hemorrhage}

J. Triscott ${ }^{1}$, S. Mercer ${ }^{2}$, B. Dobbs ${ }^{3}$, P. G. Tian ${ }^{3} .{ }^{1}$ Alberta Health Services, Edmonton, AB; ${ }^{2}$ University of Newfoundland, St. John's, NL; ${ }^{3}$ University of Alberta, Edmonton, AB.

Enoxaparin is used for anticoagulation usually without requirements and capability for laboratory monitoring. Its dosage in severe chronic renal disease needs to be reduced. However, its dosage in the elderly is not required to be lowered. This raises concerns since the elderly could have rapidly fluctuating renal functions which require immediate 
adjustments in drug dosage. We will report a case of spontaneous retroperitoneal hemorrhage in an elderly on dose-appropriate enoxaparin.

Case Report: An 81-year-old lady with chronic renal failure was admitted for diagnostics on falls/pain. She also had a pulmonary embolism four months prior to admission for which she had been treated and maintained on enoxaparin ( $1 \mathrm{mg} / \mathrm{kg}$ subcutaneously b.i.d.). Her other medications included aspirin. On admission, her laboratory results were consistent with chronic renal failure and anemia. During hospitalization, she developed vomiting, diarrhea, and melena. Abdominal CT scan showed a large retroperitoneal hematoma. We aggressively managed the ensuing hypotension and acute renal failure. We also discontinued enoxaparin and aspirin. Supportive care was provided in the intensive care unit until her renal function recovered.

Low-molecular-weight heparin (LMWH; e.g., enoxaparin) is frequently prescribed in geriatric patients. It is preferred over unfractionated heparin because LMWH does not need coagulation monitoring, is administered with ease, and is associated with decreased hospitalization cost. However, the use of enoxaparin in the elderly population poses some safety concerns. When the elderly patient is stable, enoxaparin dosing could be maximally effective; but the same dosing could rapidly become harmful when kidney function deteriorates. With vigilance, the dose could be decreased immediately during compromised renal functions; however, the risk of thrombosis would consequently increase.

The safety of enoxaparin in the elderly needs to be established.

\section{Automatic Self-Transcending Meditation Improves Heart Rate Variability in Late-Life Depression}

A. Arena ${ }^{1}$, P. Maldeniya ${ }^{1}$, E. Ionson ${ }^{1}$, A.M. Burhan ${ }^{1}$, S. Wetmore $^{1}$, A. Vasudev ${ }^{1}$, R. Newman ${ }^{2}$. ${ }^{1}$ University of Western Ontario, London, ON, Canada; ${ }^{2}$ Art of Living Foundation, Santa Barbara, CA, USA.

Current estimates of the prevalence of late-life depression (LLD) in Canada have been found to be as high as $16 \%$ and are expected to increase further as we face a more aged population. Evidence has shown that LLD is associated with a three-fold increased risk of cardiovascular disease, which may be mediated by autonomic dysfunction. Further complicating this relationship is the adverse effect some antidepressants have on autonomic tone. Therefore the aim of this study is to assess the cardiovascular benefits of a category of meditation referred to as automatic self-transcending meditation (ASTM) in LLD.
We present preliminary results $(\mathrm{N}=20)$ from an ongoing single-centre, single-blind, longitudinal RCT assessing the effects of a 12-week augmentation program of ASTM vs. treatment as usual (TAU) on heart rate variability (HRV), an autonomic parameter. Participants between 60 and 85 years of age with an Axis I diagnosis of mild to moderate LLD were randomized to the ASTM $(\mathrm{N}=9)$ or TAU $(\mathrm{N}=11)$ study arms. ASTM training was administered on four consecutive days ( $2 \mathrm{hrs} /$ day), followed by weekly $1 \mathrm{hr}$ follow up sessions for 11 subsequent weeks. ECGs were completed at baseline (week 0) and postintervention (week 12).

Thus far, the findings are promising; although only approaching significance at the current sample size, paired $t$-tests identify improvements in low-frequency HRV with ASTM training $(p=.06)$ as compared to TAU $(p=.6)$. These preliminary results suggest that ASTM may improve autonomic tone in LLD.

The study is ongoing (target $\mathrm{N}=96$ ) and will require further analysis.

If the results continue to show the effectiveness of ASTM in improving autonomic parameters, such a treatment could be readily adapted into routine clinical care.

\section{Quality Assurance in the Diagnosis of Dementia: Essential Physical Examination Components}

G.A.W. Heckman ${ }^{1}$, P. Stolee ${ }^{1}$, V. Boscart ${ }^{2}$, L.M. Hillier ${ }^{3}$, L. Lee ${ }^{4}$, F. J. Molnar ${ }^{5}$, D. Seitz ${ }^{6}{ }^{1}$ University of Waterloo, Waterloo, ON; ${ }^{2}$ Conestoga College, Kitchener, ON; ${ }^{3} \mathrm{St}$. Joseph's Health Care London, London, ON; ${ }^{4}$ Centre for Family Medicine Family Health Team, Kitchener, ON; ${ }^{5}$ Regional Geriatric Program of Eastern Ontario, Ottawa, ON; ${ }^{6}$ Queen's University, Kingston, ON.

Primary care memory clinics (PCMCs) are being established in Ontario to enhance system capacity for dementia care and efficient integration with specialized services. Preliminary evaluations are favourable, though variability in documentation of the physical examination (PE) suggests the need for Quality Indicators (QI) specific to this domain. While dementia diagnostic guidelines recommend $\mathrm{PE}$, opinion varies regarding which manoeuvres are essential.

PCMC physicians and affiliated specialists were surveyed to rate the importance of PE manoeuvres as essential, discretionary, or unnecessary to the diagnostic process. Participants were asked to identify who should perform specific PE manoeuvers.

Ninety surveys were completed (78/112 PCMC physicians, 9/23 specialists, and 3 unspecified). Most considered the assessment of orthostatic vital signs as essential and the responsibility of allied health professionals. 
Assessment of nutritional state and hygiene was deemed essential and the responsibility of referring or PCMC physicians. General PE manoeuvers were considered discretionary or unnecessary, and the responsibility of referring physicians. Gait assessments was considered essential. Specialists were significantly more likely than PCMC physicians to consider examination for facial asymmetry, extra-ocular movements, and pyramidal and extrapyramidal signs as essential. General PE was considered the purview of the referring physician. Specialists considered most neurological examination manoeuvres the purview of PCMC physicians, who generally considered these the responsibility of referring physicians. Time constraints were cited as important barriers to PE.

This study identified PE manoeuvers considered important to the assessment of patients referred to a PCMC.

A tiered approach to the PE of patients seen in PCMCs may be warranted, based on a shared understanding of roles and responsibilities of clinicians involved, and considering potential training requirements.

\section{The Geriatric Certificate Program: Building a Competent Workforce to Meet the Needs of the Aging Population}

S. Marr ${ }^{1}$, A. Patel ${ }^{2}$, D. Jewell ${ }^{3}$, L. M. Hillier ${ }^{4} .{ }^{1}$ McMaster University, Hamilton, ON; ${ }^{2}$ Regional Geriatric Program Central, Hamilton, ON; ${ }^{3}$ Regional Geriatric Program and Juravinski Research Centre, Hamilton, ON; ${ }^{4}$ St. Joseph's Health Care London, London, ON.

Geriatrics is an increasingly challenging field of practice in which there are limited opportunities for continuing education. The Geriatric Certificate Program was developed for practising regulated and unregulated health-care providers to build capacity related to geriatric assessment, behaviour management, and geriatric best practices. This study provides a preliminary evaluation of this program, examining impact on self-reported changes in knowledge, skills, and competence.

All graduates completed an on-line evaluation survey upon completing program requirements.

A total of 189 individuals are registered in the program, including nurses (30\%), allied health professionals $(19 \%)$, other regulated professionals $(7 \%)$, unregulated health professionals $(19 \%)$, and physicians $(3 \%)$. Twenty individuals have graduated to date; 16 completed a survey. Mean ratings (5-point scale) reflect that the program was very relevant to clinical practice $(4.1 \pm .72)$ and very useful in enhancing clinical practice $(4.1 \pm .62)$. The majority of graduates $(75 \%)$ reported that they are now more competent and more comfortable in providing geriatric care; $94 \%$ reported that the quality of care they provide has improved (69\%, "much improved"; $25 \%$ "improved"). The program impacted the acquisition of new knowledge $(81 \%)$ and skills $(75 \%)$ "very much" or "to a great extent." Self-reported knowledge transfer included increased use of standardized tools, use of evidence-based strategies for managing responsive behaviours, and more comprehensive care planning. Program and travel costs in the absence of financial support from employers was identified as challenge.

This program provides a significant opportunity for increasing capacity in core competencies to advance the use of best practices for geriatric care across disciplines.

This program will develop a workforce that is better prepared and supported to meet the needs of the aging population.

\section{Development of a Clinical Tool for the Detection of Hypovitaminosis D Among Community-Dwelling Older Adults}

C. Annweiler, A. Kabeshova, B. Fantino, O. Beauchet. University of Angers, Angers, France.

Universal vitamin D supplementation is not desirable. The determination of serum vitamin $\mathrm{D}$ status remains yet much more expensive than corrective supplementation. To rationalize health costs, our objective was to develop and to test a clinical diagnostic tool for the identification of older community-dwellers with hypovitaminosis D without using a blood test.

A total of 1,924 community-dwelling volunteers aged $\geq 65$ years without vitamin D supplements were recruited in this cross-sectional study. A set of clinical variables (age, gender, living alone, individual deprivation, body mass index, undernutrition, polymorbidity, number of drugs used daily, psychoactive drugs, biphosphonates, strontium, calcium supplements, falls, fear of falling, vertebral fractures, Timed Up\&Go test, walking aids, lower-limb proprioception, handgrip strength, visual acuity, wearing glasses, cognitive disorders, sad mood) was recorded from standardized questionnaires and medical examination at the time of serum 25-hydroxyvitamin D(25OHD) measurement. Hypovitaminosis D was defined as serum 25OHD $\leq$ $75 \mathrm{nmol} / \mathrm{L}, \leq 50 \mathrm{nmol} / \mathrm{L}$ or $\leq 25 \mathrm{nmol} / \mathrm{L}$. The whole sample was separated into training and testing subsets to design, validate and test an artificial neural network (multilayer perceptron, MLP).

In total, 1,729 participants $(89.9 \%)$ had $25 \mathrm{OHD} \leq$ $75 \mathrm{nmol} / \mathrm{L}, 1,288(66.9 \%)$ had $25 \mathrm{OHD} \leq 50 \mathrm{nmol} / \mathrm{L}$, and 525 $(27.2 \%)$ had $25 \mathrm{OHD} \leq 25 \mathrm{nmol} / \mathrm{L}$. MLP using 16 clinical variables was able to diagnose hypovitaminosis $\mathrm{D} \leq 75 \mathrm{nmol} / \mathrm{L}$ with accuracy $=96.3 \%$, area under curve $(A U C)=0.938$, and $\kappa=79.3$ indicating almost perfect agreement. It was also able 
to diagnose hypovitaminosis $\mathrm{D} \leq 50 \mathrm{nmol} / \mathrm{L}$ with accuracy $=$ 81.5, AUC $=0.867$ and $\kappa=57.8$ (moderate agreement); and hypovitaminosis $\mathrm{D} \leq 25 \mathrm{nmol} / \mathrm{L}$ with accuracy $=82.5, \mathrm{AUC}=$ 0.385 and $\kappa=55.0$ (moderate agreement).

We developed an algorithm able to detect, from 16 clinical variables, hypovitaminosis D with almost perfect agreement among older community-dwellers taking no vitamin D supplements.

Such an inexpensive tool will undoubtedly help clinicians in decisions to supplement their patients without routinely resorting to an expensive blood test.

\section{Engaging Patients and Caregivers in the Prevention and Detection of Delirium in the Community}

M. De Luca ${ }^{1}$, L. Mallet ${ }^{1}$, S. Sereda ${ }^{2} .{ }^{1}$ Université de Montréal, Montreal, QC; ${ }^{2}$ MUHC-Royal Victoria Hospital, Montreal, QC.

Although delirium is a common and serious geriatric syndrome among the elderly, it is often unrecognized. Prevalence of delirium in community-dwelling older adults over 85 years of age is approximately $14 \%$. The goal of this pilot study was to increase knowledge of delirium in community-dwelling elderly and their caregivers.

Multiple strategies were used in the aim of increasing knowledge on delirium. First, an educational pamphlet was developed and made available to elderly persons and their families. In addition, a 20-minute PowerPoint presentation was presented to small groups, with follow-up discussion. A pre and post test measuring knowledge levels was administered in order to measure impact. A brief survey was also conducted to obtain feedback on the quality and understanding of the content.

A total of three presentations were given with 16 participants, mean age of 53 years. A total of 12 questions were administered in the pre and post test questionnaire. Overall, participants scored higher on the post test. Feedback from participants revealed that they found the presentation and pamphlet useful in learning about delirium, especially since many participants had no previous knowledge on the subject.

The pilot study revealed that community-dwelling elders and their caregivers were not familiar with delirium. They described gaining valuable knowledge and felt the teaching methods were helpful. To further our understanding of the impact of these interventions, this pilot project will be continued in the community by 4 th year pharmacy stduents with a larger group of participants.

The results support the need for education among family members and caregivers in the community setting. Focus should be on the prevention, identification, and management of delirium.

\section{Presentation of a Newly Developed Visual Acuity Screening Tool for Older Institutionalized Seniors with Dementia}

H. Kergoat, C. Law, S. Chriqui, M.-J. Kergoat, B.-S. Leclerc. Université de Montréal et Institut universitaire de gériatrie de Montréal, Montreal, QC.

Our recent data have shown that eyecare services offered to older institutionalized seniors with dementia are not optimal. We present here the development of a visual acuity (VA) screening tool aimed at improving eyecare services for those individuals.

Some two decades ago, the World Health Organization developed a tool to screen VA in individuals from developing countries. The tool comprised tumbling E's presented on plastic cards. Researchers have since used this tool for screening VA in older institutionalized seniors, including those with dementia, even if it has never been validated in dementia. We have therefore developed a new tool that will be presented at this meeting. Testing is currently being performed in volunteer institutionalized seniors $\geq 65$ y.o. with cognitive impairment.

The tool has been developed on a retinal-display iPad to provide repeatable, high-resolution, high contrast optotypes. It contains 3 kinds of optotypes (letters, numbers, tumbling E's), 4 symbols per optotype and 3 levels of VA. It allows testing of each eye at 2 working distances and gives the time taken for each test. Each symbol is displayed one at a time in the centre of the screen, from the largest to the smallest one, in a random sequence for each target size.

This new tool is successfully being used in older institutionalized seniors with mild to severe dementia. Its application for that population has therefore been shown feasible. Within a year, we will have collected enough data to determine if the tool is valid for that population.

If the tool is valid, then long-term care units will benefit from a VA screening tool to help determine those residents most needing an oculovisual examination.

55. Prevalence of Neuropsychiatric Symptoms in Long-Term-Care Residents with Dementia across the Circadian Cycle

M. Wilchesky ${ }^{1}$, S. Ballard ${ }^{1}$, B. Gore ${ }^{1}$, O. Lungu ${ }^{1}$, M.-A. Bruneau $^{2}$, P. Voyer $^{3}$, P. Landreville ${ }^{3}$, R. Verreault ${ }^{3}$, E. Kröger $^{4}$, N. Champoux ${ }^{5}$, J. Monette ${ }^{6}$. ${ }^{1}$ Donald Berman Maimonides Centre for Research in Aging, Montreal, QC; ${ }^{2}$ Université de Montréal, Montreal, QC; ${ }^{3}$ Université Laval, Quebec, QC; ${ }^{4}$ Centre d'excellence sur le vieillissement de Québec, Quebec, QC; ${ }^{5}$ Institut Universitaire de Gériatrie de Montréal, Montreal, QC; ${ }^{6}$ Jewish General Hospital, Montreal, QC. 
Neuropsychiatric symptoms (NPS) are behavioural and non-cognitive manifestations of dementia that are highly prevalent among residents in long-term care (LTC), increasing costs and burden of care for this vulnerable population. A common clinical phenomenon observed among people with dementia is "sundowning syndrome," or the emergence or exacerbation of NPS in the late afternoon, evening, or at night. Very few studies, however, have assessed NPS prevalence in LTC residents with dementia as a function of time of day.

Frequency and severity of NPS were assessed over a 2 -week period using the Neuropsychiatric Inventory - Nursing Home version. NPS information for 97 LTC residents with dementia was obtained from frontline nursing staff providing their direct care during the day, evening, and night shifts. A total of 238 staff interviews were conducted, and complete triad data (across all shifts) was obtained for 59 residents.

NPS prevalence was $66.1 \%, 71.2 \%$, and $42.4 \%$ during day, evening, and night, respectively.

Agitation/aggression and irritability were the most prevalent symptoms, even after accounting for sleep dysregulation. The percentage of residents exhibiting more than four NPS increased significantly from $13.6 \%$ (day) to $20.34 \%$ (evening) $(p<.01)$, suggesting the presence of sundowning syndrome. Although depression was prevalent in about $15 \%$ of residents at all times of day, its presence was significantly associated with anxiety (all times), aggression/ agitation (day and evening), irritability (day), and delusions and hallucinations (night).

Sundowning syndrome manifests itself as an increase in the number of NPS observed within a given resident rather than as an increase in overall NPS prevalence. Depression is strongly associated with multiple NPS.

Our results highlight the importance of considering time of day when evaluating NPS in LTC residents.

\section{A Cohort of Alzheimer Disease and Dementia Cases from Quebec Administrative Data: Prevalence and Incidence between 1998 and 2012}

E. Kröger ${ }^{1}$, R. J. Laforce ${ }^{1}$, L. Rochette ${ }^{2}$, É. Pelletier ${ }^{2}$, V. Émond ${ }^{2}$, C. Bocti ${ }^{3} .{ }^{1}$ Université Laval, Quebec, QC; ${ }^{2} \mathrm{INSPQ}$, Quebec, QC; ${ }^{3}$ Université de Sherbrooke, Sherbrooke, QC.

In 2011, Alzheimer's disease (AD) and dementia affected 747,000 Canadian seniors (65+). Estimates of the prevalence or incidence of dementia are frequently based on the Canadian Study of Health and Aging (CSHA, 1991-2001). However, administrative data are increasingly used for the surveillance of chronic diseases, including $\mathrm{AD} / \mathrm{dementia}$, and may add new insight into care trajectories. The present study identified a Quebec cohort of $\mathrm{AD} / \mathrm{dementia}$ cases using linked health administrative data.
This study used data linked at the Institut national de santé publique du Québec provided from 3 sources: 1) the RAMQ medical and pharmaceutical services and the registered users' files, 2) the MED-ECHO hospital discharge file and 3) the death registry. $\mathrm{AD} /$ dementia cases were identified using three definitions with different combinations of ICD dementia-related diagnostic codes from physicians or hospital discharge, with or without pharmacy claims for 4 dementia medications. Two of these definitions were validated by the Public Health Agency of Canada and one will be used for further surveilance. Estimates were age-standardized.

The study included 1.3 million persons aged $65+$. In $2011-12,6.8 \%$ to $10 \%$ among them were identified as cases, depending on the definition. Prevalence was $15 \%$ to $18 \%$ higher among women than men and rose exponentially in older age groups. About $1 \%$ to $2.5 \%$ of persons aged 65 to 69 years were identified as cases, compared to $27 \%$ to $35 \%$ for people $85+$ years. Incidence rates varied between 16.5 and 21.7 per 1,000 person-years depending on the case definition. In this study, highest incidence rates were measured in 2006-07 for all case definitions.

In Quebec data, physician claims were the main source for case ascertainment, followed by hospital data. The observed measures of prevalence and incidence are below those that can be extrapolated from the CSHA and Canadian demographics, possibly due to underreporting in administrative data. Different data sources for incidence and prevalence estimates are used in Canada and their interpretation can be challenging.

Ongoing surveillance from health administrative data in Quebec is feasible and useful for research, policy, and program guidance.

\section{Potentially Inappropriate Medication Use in the Elderly: A 2-Year Review of Comprehensive Geriatric Assessments}

E. Elghol, L. Charles, B. Dobbs, O. Babenko, J. Triscott, P. G. Tian. University of Alberta, Edmonton, AB.

Certain drugs have been linked to adverse events and poor outcomes in the elderly. These drugs, called Potentially Inappropriate Medications (PIM) by the American Geriatrics Society, should be avoided in the elderly population, through treatment with safer drugs and/or nondrug approaches. In this study, we will determine the (1) prevalence of PIM use among patients referred for comprehensive geriatric assessments; (2) most common PIMs used; (3) extent comprehensive geriatric assessments addressed PIM use.

Design: This was a cross-sectional study involving chart reviews of 200 patients (65 years and older) seen for 
Comprehensive Geriatric Assessments at the Glenrose Rehabilitation Hospital in 2012-2013.

Procedure: We reviewed the use of PIMs, as defined by the 2012 American Geriatrics Society Updated Beers Criteria. Specifically, we noted the use of Medications to Avoid in Older Adults Regardless of Disease or Conditions (Group 1) and Medications Considered Potentially Inappropriate When Used in Older Adults with Certain Diseases or Syndromes (Group 2).

The patients had a mean age of $79 \pm 8$ years. The prevalence of PIM use was $49.5 \%(\mathrm{~N}=98)$. For Group-1 medications, $45.5 \%(\mathrm{~N}=91)$ of patients used at least one PIM. For Group-2 medications, $31 \%(\mathrm{~N}=62)$ of patients used at least one PIM. The most common Group-1 PIMs used were Zopiclone, Benzodiazepines, and pain medications. The most common Group-2 PIMs used were Benzodiazepines, Zopiclone and Selective Serotonin Reuptake Inhibitors. Of the 98 patients who used PIMs, 48\% $(\mathrm{N}=47)$ had their medications stopped, tapered, adjusted, decreased, or reviewed as a result of Comprehensive Geriatric Assessments.

The 2012 Beers Criteria is a useful guideline for safe drug use and monitoring in the elderly.

PIM use in the elderly is common and needs to be addressed.

\section{Implementation of Capacity-Building Strategies in Long-Term Care to Reduce Potentially Preventable Emergency Department Visits}

G. Naglie, A. Uy, M. Hohmann, A. Berall, A. M. Shin, J. Goodhand, C. Anderson, P. Katz, J. Karuza. Baycrest Health Sciences, Toronto, ON.

Long-term care (LTC) residents have high acute-care utilization rates and face elevated risks when transitioning between LTC and acute care. The objective of this study was to evaluate the initial implementation of components of the Interventions to Reduce Acute Care Transfers (INTERACT) program as part of an innovative strategy to improve the capacity of staff to handle acute change in LTC residents and reduce potentially preventable emergency department (ED) visits and hospitalizations from Baycrest.

INTERACT is a quality improvement program to improve the identification, evaluation and communication of changes in LTC residents' status. The initial implementation methods included primarily education and reminders. The evaluation used a mixed-methods approach including electronic health record reviews, audits of communication tools, tracking of ED visit and hospitalization rates, and surveys and focus groups.
Preliminary findings reveal that initial implementation methods have not resulted in sustained changes in practice. Survey data collected at the onset of implementation indicated that one-third of the LTC units were in the precontemplation phase of change and two-thirds of the units were in the contemplation phase of change. Focus group data from point of care staff noted that the communication tools were too time-consuming and did not facilitate the preferred communication. In addition, multiple barriers have been encountered in measuring potentially preventable ED visits and hospitalizations.

Our findings indicate that education and reminders alone are not sufficient for implementing sustained changes in practice related to better identifying and managing changes in status in LTC residents.

To ensure greater success in implementing capacity building strategies in LTC, future efforts will focus on driving forces for change such as urgency for change, aligning with organizational systems and strategies, and committed change agents.

\section{An Interdisciplinary Nursing Home Intervention to Reduce Inappropriate Medication Use Among Residents with Severe Dementia: OptimaMed}

E. Kröger ${ }^{1}$, P. Voyer $^{1}$, M. Morin ${ }^{1}$, A. Giguère ${ }^{1}$, M. Aubin ${ }^{1}$, P. Durand ${ }^{1}$, M. Wilchesky ${ }^{2}$, J. Monette ${ }^{2}$, N. Champoux ${ }^{3}$, M. Arcand ${ }^{4}$. ${ }^{1}$ Université Laval, Quebec, QC; ${ }^{2}$ McGill University, Montreal, QC; ${ }^{3}$ Université de Montréal, Montreal, QC; ${ }^{4}$ Université de Sherbrooke, Sherbrooke, QC.

Nursing home $(\mathrm{NH})$ residents with severe dementia often receive multiple medications. With disease progression care goals shift from curative or preventive to comfort care and consequently medications have to be reviewed, adjusted, or discontinued, because of reduced life-expectancy or changes in their harm-benefit ratio. Few studies evaluated interventions to achieve this goal. The objective of this pilot study was to evaluate the feasibility of an inter-professional intervention to optimise medication use in $\mathrm{NH}$ residents with severe dementia.

Based on a literature review and a multidisciplinary Delphi panel, lists of mostly, sometimes, or rarely appropriate medications and elements of successful interventions were identified. The lists were tailored for a NH pilot study. Between April and November 2014 a 4-month intervention was led in $3 \mathrm{NH}$ in Quebec, Canada. The families of participating residents received an information leaflet on optimal medication use in severe dementia. Nurses, pharmacists, and physicians of the $\mathrm{NH}$ participated in two 90-minute continuous education (CE) sessions. For each participant the pharmacist performed a medication review using the lists and then discussed 
recommendations with nurses and physicians. A study nurse observed comfort and agitation levels of participants using the Cohen-Mansfield and the PACSLAC-F scales during the study period.

93 residents were eligible and 48 participated; 7 residents died before or during follow-up and 41 were observed over 4 months. 38 health professionals participated in the CE sessions. Medication lists were well accepted and the study nurse was present at the discussions about medication changes. Families' and health professionals' comments provide opportunities to improve information material and the tailored lists. Some changes in medication use were observed but levels of agitation and comfort did not change noticeably.

An interdisciplinary $\mathrm{NH}$ intervention to optimise medication use in residents with severe dementia is feasible. The three NH in Quebec City were interested in opportunities and tools facilitating improved medication use. Results from the pilot study need to be repeated in a larger trial and education or information to families should be a focus of the study.

A literature review and an expert consensus provided the elements for a feasable intervention to optimize medication use. A cluster randomized trial should validate medication outcomes, generalizability, and patient or family outcomes of this intervention.

\section{Pilot Study on the Feasibility of Establishing an Intervention Aimed at Preventing Functional Decline of Patients Hospitalized in Geriatric Assessment Units (GAU)}

M.-J. Kergoat ${ }^{1}$, J. Rousseau ${ }^{1}$, P. Nguyen ${ }^{1}$, F. Dubé ${ }^{1}$, M.-È. Ringuet $^{1}$, A. Bolduc ${ }^{1}$, B.-S. Leclerc ${ }^{2} .{ }^{1}$ Université de Montréal, Montreal, QC; ${ }^{2} \mathrm{CSSS}$ de Bordeaux, Montreal, QC.

Background: Programs for preventing functional decline in the elderly hospitalized in acute care facilities are suggested in the literature and in clinical guidebooks. In general, they are poorly detailed and primarily involve physiotherapists. Rehabilitation professionals affiliated with the IUGM GAU have developed a physical reconditioning program (SPRINT) that can be adapted to the functional profile of patients admitted to GAU. This program has 4 different levels and includes ad-lib repetitions of motor activities prescribed after an evaluation of functional capabilities, ranging from transfer to a chair (level 1) to walking (level 4). The program engages the patient and enlists the contribution of medical personnel and natural caregivers with daily interactions with the patient.

Purpose: Collect preliminary information on the implementation process of SPRINT in order to determine the conditions necessary for rolling out SPRINT to GAU.
Methods: The project, currently under way, will last one year at the IUGM GAU and includes 4 stages: preparation, pre-intervention, intervention, and post-intervention.

Results: Amongst the 47 patients admitted to the GAU to date, 18 patients (61\% women) accepted to participate. They were aged $80.5 \pm 8.2$ years, had an MMSE score of $26.4 \pm 3.4$ and participated in SPRINT for $19.1 \pm 9.3$ days. Only SPRINT levels 2 and 3 were prescribed to recruited patients. Patients did an average of 0.1 to 4.1 exercises/day. Exercises were done with a nurse (49\%), a doctor (21\%), or alone by the participant $(18 \%)$; natural caregivers only participated in $4 \%$ of cases.

Conclustions: Qualitative data concerning factors that facilitated and perceived barriers will be collected and analysed to facilitate the successful implementation of the intervention.

\section{Prescribing Cascade Game for Pharmacy Students}

L. Mallet. University of Montreal, Montreal, QC.

Although associated with adverse drug events in the elderly, prescribing cascades are often not recognized in clinical practice. The objective of this prescribing cascade game was engage students in learning how to prevent, detect, and understand prescribing cascades.

This activity was part of a 3 -credit course in the geriatrics pharmacotherapy course at the Faculty of Pharmacy at the University of Montreal. Four different prescribing cascades that were detected in clinical practice were used. Each cascade contained four elements (medication-side effectmedication-side effect). Students were divided into 16 groups with two students per group. Each group selected one card on which one of the elements was written: either the name of a medication, or a side effect. All 16 groups were then asked to work together to reconstruct the four different prescribing cascades. Once all four elements of the four prescribing cascades were identified, they were asked to explain how these cascades took place. A 5-point Liker scale was used to evaluate the perception of the students regarding this activity.

32 students participated in the game. It took 15 minutes for the students to get organized and reconstruct the four prescribing cascades. In general, students appreciated this activity. The majority mentioned that having to find the different element of the cascade raised their awareness concerning the detection of future cascade.

This activity was used to understand, apply, and retain information on prescribing cascades. Students actively participated in this activity. They were able to discover the four different elements of their respective cascade and to explain them using ptharmacokinetics, pharmacology, and pharmacodynamics principles. 
The prescribing cascade game was received positively by the students. It will now be part of the geriatrics pharmacotherapy course.

\section{Tablet Training for Seniors: The iLearn iLive Well Program}

S. Marr ${ }^{1}$, J. Firman ${ }^{1}$, L. Panamsky ${ }^{1}$, P. Cowman ${ }^{1}$, R. Weldrick $^{1}$, J. Waisglass ${ }^{1}$, L. M. Hillier ${ }^{2}$, A. Patel ${ }^{3}$, D. Jewell ${ }^{4}$. ${ }^{1}$ McMaster University, Hamilton, ON; ${ }^{2}$ St. Joseph's Health Care London, London, ON; ${ }^{3}$ Regional Geriatric Program Central, Hamilton, ON; ${ }^{4}$ Regional Geriatric Program and Juravinski Research Centre, Hamilton, ON.

Internet use among older adults has the potential for significant social and health outcomes. However, few seniorfocused and senior-friendly training opportunities exist. The purpose of this study was to evaluate the feasibility of delivering a senior-friendly tablet training program and its impact on tablet ownership, sustained use, and confidence over time.

Twelve seniors completed the iLearn iLive Well tablet training program (six 2-hour weekly sessions) to teach participants how to operate a tablet including e-mail and internet use; availability and use of apps; and viewing, taking, and sending photographs. Participants completed pre- and post-program surveys, session reaction surveys, and 3-month follow-up interviews to assess sustained use of learned skills.

All of the sessions were well received with most of the sessions being rated as "very good" or "excellent" by over $67 \%$ of participants. At follow-up 8 of 11 interviewed participants reported that they had purchased a tablet, $63 \%$ reported using it more frequently since the end of the training program, and $88 \%$ reported being more confident in their ability to use the tablet in comparison to before the program. As a result of their tablet use, participants reported being in greater contact with friends and family than they would have been otherwise, being better informed of current affairs, being able to access to information more easily, and having a great sense of satisfaction with learning new things. One-to-one mentorship, senior-friendly training manual, learning strategies, environment, and previous computer experience facilitated their tablet learning. Identified learning barriers were mostly program-specific and modifiable.

This senior-focused tablet training program facilitated sustained tablet use.

Future studies will examine the impact of this program on perceived social isolation, loneliness, and attitudes towards computers.

\section{The Prevalence and Risk factors of Anorexia in the Korean Elderly}

M.-Y. Kim, J.-C. Kim. Seoul Medical Center, Seol, Korea.

Anorexia in the elderly often cause weight loss, therefore it is regarded as a important starting point of frailty syndrome, which is considered as a critical point on the pathway to disability and mortality. Korea is entering an aging society at the fastest speed in the world, but the prevalence and risk factors of anorexia in the Korean elderly have not been investigated.

A total of 170 men and women (30 from nursing homes, 140 from out-patient clinics) older than 60 years were recruited in this study. We evaluated the appetite with a Simplified Nutritional Appetite Questionnaire (SNAQ) and defined the anorexia subjects as those whose SNAQ score was less than 14. We also performed a comprehensive geriatric evaluation including the measurement of anthropometric data; the medical, drug, and social history; cognitive function with the Korean version of the mini mental state examination (K-MMSE); activity of daily living (ADL); the Geriatric depression scale (GDS); and the Mini-nutritional assessment (MNA).

The prevalence of anorexia was $50 \%$ in nursing home residents and $45 \%$ in ambulatory elderly patients. Comparing with non-anorexic subjects, the anorexic subjects showed lower K-MMSE score $(24.7 \pm 5.33$ vs $26.4 \pm 4.01, \mathrm{p}=.026)$ and MNA score $(10.2 \pm 2.90 \mathrm{vs}$ $11.4 \pm 2.59, \mathrm{p}=.004)$ and more frequently expressed gastrointestinal symptom such as diarrhea $(8.0 \%$ vs $1.1 \%, \mathrm{p}=.046$ ). We could not show statistical significant relationship between the anorexia and body mass index, calf circumference, Cumulative Illness Rating Scale, the number of medications, ADL and GDS score, residual teeth number and social support factors. Logistic regression analyses showed that the MMSE score was independently related to the anorexia $(p=.005)$.

The prevalence of anorexia in our study was higher than the results in the other countries, and this result may be due to the high prevalence of polypharmacy. The average number of medications in this study was $4.9 \pm$ 3.70. Because the study population was not selected by random sampling, the results of this study could not represent the anorexia prevalence of the general Korean elderly population.

We showed a high prevalence of anorexia in the Korean elderly. Because the SNAQ is regarded as the early detection tool of malnutrition and weight loss, further prospective studies are essential to determine the long-term consequences of the anorexia assessed with SNAQ. 


\section{Decision-Making Capacity Assessment (DMCA) Training for Physicians}

L. Charles, J. Parmar, B. Dobbs, S. Bremault-Phillips, O. Babenko, J. Triscott, P. Tian. University of Alberta, Edmonton, AB.

Physicians play a key role in DMCA. However, many do not feel prepared based on their residency training. They, thus, often require additional training once in practice. To address this need, we developed and administered an interactive DMCA Workshop to familiarize physicians with a DMCA model, including concepts of capacity, the protocol, documents, and case studies. In this study, we will determine the effect of the DMCA Workshop on physicians' confidence and comfort with decision-making capacity assessments.

Design: This study used a pre-test-post-test design. We administered a questionnaire before and after the Workshop. The questionnaire asked participants to rate their agreement (4-point Likert-type scale) on 15 statements regarding awareness, confidence, and understanding of core concepts of capacity.

Participants: All physicians who attended three workshops in 2014.

Intervention: A 3-hour DMCA Workshop accredited by the College of Family Physicians Canada.

There were 54 participants with an average age of 47 years. There was a significant improvement $(p<.001$, Sign Test) in the post- compared to the pre-workshop ratings for all the items. The highest positive differences were seen for awareness of legislative acts, understanding a trigger, problem-solving techniques, standardized approach, and knowledge and skill-set in regards to capacity assessments. Among the participants, those without prior DMCA training exhibited the largest change in pre- versus post-workshop ratings $(\mathrm{p}<.05)$.

The results indicate that the information provided in the workshops is valuable for physicians regardless of years worked in the current setting. The pattern of findings provides important feedback on modifications to delivering future workshops.

This study has shown that a DMCA Workshop was effective in training Family Physicians. The next step is to customize the Workshop to Family Physicians' needs.

\section{RUSHGQ Prescribing Guidelines for Short-Term Geriatric Assessment Units (GAU)}

M.-J. Kergoat ${ }^{1}$, J. Latour ${ }^{1}$, A. Bolduc ${ }^{2}$. ${ }^{1}$ Centre Hospitalier de l'Université de Montréal, Montreal, QC; ${ }^{2}$ Centre de recherche, Institut universitaire de gériatrie de Montréal, Montreal, QC.

The Regroupement des Unités de Courte Durée Gériatriques et des Services Hospitaliers de gériatrie du Québec (RUSHGQ) is a geriatric community of practice that includes health-care professionals and managers from 51 of the 61 Quebec hospitals with Geriatric Assessment Units (GAU). The RUSHGQ established a work sub-committee composed of doctors and pharmacists throughout Quebec with the purpose of establishing guidelines for medication management in GAU.

Doctors and pharmacists with experience in GAU have prepared, using evidence-based data and consensus decisions, a user guide on appropriate medication management for healthcare professionals in GAU. To date, the covered drug classes are: statins, antihypertensives, direct oral anticoagulants, sedatives/hypnotics, antidepressants/psychotropic drugs, as well as medications for urinary incontinence

Six workgroups composed of GAU doctors and pharmacists were formed to cover each of the themes; each workgroup was headed by a doctor considered by his/her peers to be a theme expert. This iterative work lasted 12 months.

The guidelines begin with a general section detailing pharmacokinetic and pharmacological changes linked to aging, specific precautions and the notion of deprescribing, which must be considered when prescribing medications to patients admitted to GAU. The typical profile of GAU patients has been described in previous work by the RUSHGQ: $\geq 80$ years old with multiple acute and chronic conditions and polymedicated. Then, specific information sheets are presented which include: (1) a summary of the indications of the medication of interest; (2) usage guidelines adapted to the geriatric population; (3) clinical cases integrating the presented concepts. Appendixes provide complimentary tools.

Prescribing guidelines presently covering 6 different themes were elaborated by expert clinicians in order to maintain/propagate good practices in medication management in GAU.

\section{Optimizing Bowel Health for Older Adults in the Rehabilitation Setting}

Wanda Kiersnowski, Carol Skanes. University Health Network, Toronto, ON.

The Geriatric Rehabilitation Program at UHN facilitates the transitions of older adults from the acute care setting to home. Participation and progress in rehabilitation is hampered at times by complications related to bowel function. The patient population includes patients with multiple co-morbidities including those with end-stage 
renal disease receiving hemodialysis. In order to better understand the prevalence of bowel-related concerns on the unit, a chart audit was conducted revealing approximately fifty percent of the patient population met the criteria for constipation as per the Rome III classification system. A review of current practices on the unit and existing documentation revealed opportunities for improvement.

The unit leadership, consisting of the Clinical Nurse Specialist, Advance Practice Nurse Educator, and Program Service Manager, developed a multi-faceted strategy to enhance the interprofessional team's ability to optimize the bowel health of patients. Components of our strategy included: implementing nursing interventions to enhance healthy bowel function, introducing the Bristol stool chart for assessment, patient education, evaluation of interventions, and team communication as well practice supports at the bedside.

Members of the interprofessional team contributed updates on best practices and participated in educational sessions with the nurses to enrich the dialogue and assist with translation to practice.

Evaluation of this important initiative included auditing documentation, communication at team rounds, and prevalence of constipation post-implementation.

Optimizing Bowel Health was a significant clinical and educational initiative that improved the interprofessional team's ability to monitor, document, and discuss priority concerns related to bowel function. This is especially important in a rehab setting where complications often lead to decreased participation, interruptions in therapy, and decreased quality of life experiences for patients.

The results demonstrated enhanced documentation, interprofessional communication, and reduction in rates of constipation.

\section{A Study of the Place-of-Death Preference amongst Chinese Nursing Home Residents in Hong Kong}

N. P. K. Law ${ }^{1}$, K. C. Choi ${ }^{1}$, C. W. H. Chan ${ }^{1}$, J. K.H. Luk ${ }^{2}$. ${ }^{1}$ The Chinese University of Hong Kong, Hong Kong; ${ }^{2}$ Fung Yiu King Hospital, Hong Kong.

Older people living in nursing homes $(\mathrm{NH})$ in Hong Kong have significant mortality. Their preference of place of death and the factors affecting their decision have not been explored. This study examined the preference of place of death and the predictors in older people living in $\mathrm{NH}$.

A cross-sectional quantitative study by face-to-face interview in $20 \mathrm{NH}$ selected by convenience sampling. A structured questionnaire was employed to collect information about attitudes of older people towards end-oflife (EOL) issues and preferences for place of death. $\mathrm{NH}$ residents aged $\geq 65$ with Abbreviated Mental Test (AMT)
Score $\geq 6$ and ability to communicate in Cantonese were recruited. AMT, Barthel Index (20) and Chinese version of the European Quality of Life-5 Dimensions (EQ - 5D) for quality of life assessment were collected. The attitudes toward EOL issues (AEOLI) were examined using a validated translated questionnaire.

$317 \mathrm{NH}$ residents (248 women and 69 men) aged 65 to 99 (mean \pm SD) $84 \pm 6.6$ were recruited. Most residents $(\mathrm{N}=$ $310,97.8 \%$ ) viewed $\mathrm{NH}$ as their own home. There were 216 (68.1\%) who wished to pass away in $\mathrm{NH}$. $\mathrm{NH}$ residents who preferred receiving care in hospital if they were severely ill without hope of recovery were less likely to wish to die in $\mathrm{NH}$ (odds $0.49, \mathrm{CI} 0.3$ to $0.81, \mathrm{p}=.005$ ). Those who agreed physician-assisted suicide if they were severely ill without hope of recovery were likely to wish to die in RCHE (odds 1.95 , CI 1.07 to $3.56, \mathrm{p}=.029$ ).

EOL services should be enhanced in NH in Hong Kong. Further studies are recommended to examine the attitudes towards dying in place in family members, $\mathrm{NH}$ staff and local public.

Many older Chinese people in Hong Kong viewed NH as their own home. More than two-thirds of NH residents wished to die in $\mathrm{NH}$.

\section{Is Filial Piety Associated with Suicidal Ideation in a Chinese Aging Population?}

X. Dong. Rush University, Chicago, IL, USA.

Suicidal ideation is a public health issue that has a significant impact at the individual, family, community, and societal levels. This study aimed to examine the association between filial piety and suicidal ideation among U.S. Chinese older adults.

Guided by a community-based participatory research approach, 3,159 community-dwelling Chinese older adults in the Greater Chicago area were interviewed in person 2011-2013. Independent variables were expectations and receipt of filial piety from the older adult's perspective. Dependent variables were suicidal ideation in the last 2 weeks and last 12 months. Logistic regression analyses were performed.

Of the 3,159 participants interviewed, 58.9\% were female and the mean age was 72.8 years. After adjusting for age, sex, education, income, medical comorbidities, and depressive symptoms, lower receipt of filial piety was associated with increased risk for 2-week suicidal ideation (OR 1.07, 95\% CI 1.03-1.11) and 12-month suicidal ideation (OR 1.07, 95\% CI 1.04-1.11). The lowest tertiles of filial piety receipt was associated with greater risk for 2-week suicidal ideation (OR 1.95, 95\% CI 1.12-3.38) and 12-month suicidal ideation (OR 2.17, 95\% CI 1.35-3.48). However, no statistically significant associations were found between 
filial piety expectations and suicidal ideation in the last 2 weeks or in the last 12 months.

This study suggests that filial piety receipt is an important risk factor for suicidal ideation among U.S. Chinese older adults.

However, future longitudinal studies are needed to quantify the temporal association between filial piety and suicidal ideation.

\section{The Importance of Not Judging a Book by Its Cover: The Need to Specifically Evaluate Seniors for Sarcopenia}

A. Juby, C. Davis, S. Minimaana, M. Cree. University of Alberta, Edmonton, AB.

Prevalence of sarcopenia increases with age. Sarcopenia is associated with higher rates of drug side effects, hospitalacquired infections and functional decline. Sarcopenia subtypes are defined by the European Working Group of Sarcopenia in Older People (EWGSOP), based on presence/ absence of low muscle mass, plus/minus low muscle strength or low performance.

Participants are independently mobile, communitydwelling seniors. Baseline assessments included dual energy Xray absorptiometry (DXA) body composition (BC) analysis, grip strength (dynamometer), and gait speed (10 metre walk test). BC provided appendicular lean mass/ height2 (aLM/ht2) and percentage body fat. Data evaluated per EWGSOP guidelines. Low grip strength and gait speed, with normal aLM/ht2 were classified as "weak," to differentiate them from normal. Prescaropenics had only low aLM/ht2, sarcopenics had low aLM/ht2 plus one abnormal level in one of the other parameters, and severe sarcopenics had abnormal levels in all parameters. Obesity was defined by DXA BC percentage fat of $>40 \%$ (women), $>28 \%$ (men).

39 participants were evaluated: 32 women, 7 men; average age 75.9 years (67-90); average MoCA 25.5. EWGSOP classification of the 32 women: 9 normal; 2 presarcopenia; 2 presarcopenic obesity; 1 sarcopenia; 1 severe sarcopenia; 1 severe sarcopenic obesity; 3 sarcopenic obesity; 10 obese; 1 normal "weak"; 2 obese "weak." Of the 7 men: 2 normal; 1 sarcopenia; 3 sarcopenic obesity; and 1 obese. The subgroups were comparable for age. Baseline BMI was 27.5 (18.8-37.5) and BMI did not discriminate the body types.

In these independent, highly functioning seniors, there was a surprising diversity of body composition.

BMI alone was of no use in classifying body type. The EWGSOP classification is useful to stratify an outwardly homogenous group of seniors.
71. Riding the Silver Tsunami in Primary Care: Optimizing Geriatric Polypharmacy by Implementation of an Automated Medication Review System at the St Michael's Academic Family Health Team

D. Wang, M. Lam-Antoniades. University of Toronto, Toronto, ON.

Polypharmacy is becoming increasingly prevalent in our aging population, due to the need to treat various disease states that may develop with age. Geriatric polypharmacy has been correlated with a significant patient safety issue, due to its association with higher likelihood of nursing home placements, morbidity, hospitalization, and death. Primary care teams represent the first point of contact, and are well positioned to address this phenomenon. The purpose of this project was to develop an automated medication review process in high-risk patients (defined as those 75 years of age or older and on 7 medications or more) by optimizing collaborative process with a clinical pharmacist and taking advantage of the capabilities of the electronic medical record (EMR).

Patients were identified via a search on the EMR and flagged for a medication review. An optimal process for referral and communication between providers was developed using an iterative approach, taking into consideration feedback from the team members and patient reactions. Data were also collected on the types of pharmacist interventions and the types of medications changed.

We developed a process using electronic messaging and coordinated MD-pharmacist visits. The total number of medications per patient was reduced, and the medications were adjusted based on the current geriatric literature.

Patients were more likely to agree to a face-to-face meeting if the idea was introduced by their regular provider. Co-ordination of pharmacistand MD appointments led to more efficient implementation of pharmacist recommendations. Inferentially, by optimizing polypharmacy, harms of negative clinical outcomes would be reduced.

Using EMR capabilities, a workable and sustainable process for performing geriatric polypharmacy reviews in the primary care setting was developed, and can easily be disseminated to other sites.

\section{Painful Non-Healing Wounds in an Elderly Patient: Could It Be Angiosomal?}

C. Ott ${ }^{1}$, A. Moser ${ }^{1}$, G. Papia ${ }^{2} .{ }^{1}$ Baycrest Hospital, Toronto, ON; ${ }^{2}$ Sunnybrook Hospital, Toronto, ON.

Case Report: 97-year-old female presented with two painful ulcers on her left foot. One of the ulcers was determined to be an osteomyelitis. 
3 months into treatment of these non-healing, painful wounds, she developed a third painful wound on her left calf.

Her wounds treated with topical dressings and antibiotics. Pain required multiple opiods with limited success.

Seen by vascular surgery 6 months into treatment. Problem determined to be angiosomal. Treated with angioplasty. Ankle brachial ratio was increased from 0.28 to 0.57 . Rapid healing over next few months ensued. Two years later, patient is walking around the nursing home.

Vascular surgery consultation with computed tomography angiogram should be considered earlier in treatment to decrease morbidity, improve quality of life, and lessen pain symptoms.

Advanced age is not a reason to not consider consultation.

\section{Geriatric Medically Complex Clinic}

L. Murray, N. Didyk. St. Mary's General Hospital, Kitchener, ON.

St. Mary's General Hospital has been awarded a demonstration project for the development of a Geriatric Medically Complex Clinic (GMCC). BRIDGES, based out of the University of Toronto, has been engaged for project evaluation. Geriatric patients with conditions such as dementia, cognitive decline, delirium, falls, fractures, multiple co-morbidities (CHF, COPD and diabetes), and chronic pain can be compounded by cognitive decline, caregiver stress, frailty, and polypharmacy. When geriatric patients have suboptimal care or are unable to access traditional primary care providers, they will resort to crisis teams and emergency departments (EDs), presenting a significant cost to the health system.

For the ED the GMCC specifically targets those who have suboptimal access/utilization of traditional primary care providers and sees the patient within 72 hours. The GMCC has partnered the community to ensure urgent referrals are also seen within 72 hours.

The GMCC team follows patients through their point of referral (i.e., ED, community, or hospital stay), with the guidance and support of geriatric medicine specialists or primary care practitioner. The team provides a focused comprehensive assessment and targeted inter-professional intervention, intensive case management across the spectrum of care, and facilitation of successful transitional care from hospital to the community. For those who are unable to leave their homes, management of chronic diseases through partnership with Primary Care supports the patient in the community, providing patient-centered care across the continuum.

Through the use of an inter-professional team, patients/ caregivers will have system navigation regarding health-care options, and access to timely community supports/services to manage their health-care needs.
The goal is to improve patient outcomes, access community supports to successfully manage in the community post a hospital interaction, and reduce healthcare costs.

\section{Evaluation of Family Structure of the Highly Vulnerable Elderly}

M. Terassi, E. S. Rossetti, M. S. Zazzetta, S. C. I. Pavarini, K. Gramani-Say. Universidade Federal de São Carlos, São Carlos, SP, Brazil.

The objective of this study was to evaluate the family composition of elderly caregivers that were identified in the Basic Health Unit localized in an area of high social vulnerability.

Seventy-three elderly caregivers participated in the study. Collection took place from May to October 2014. The genograms were made through interviews in their homes. All ethical principles were observed.

The mean age was 70.35 years ( $\mathrm{SD} \pm 8.5), 58$ elderly women and 15 men. Mean education was 2.3 years. We found the average of three people per household and 5.53 children per individual. Most seniors reported normal bonding with family. The average income of the elderly was 0.93 minimum wage and average family income was 2.3 minimum wage.

The genogram is a good tool to investigate characteristics and familial relations, revealing the interviewed's non-verbal language; however, these relationships change over time.

Most of the patients reported normal bonding with family. The genogram has proved an effective tool for assessing the family structure of the elderly in a Basic Health Unit and can be used as a mechanism to improve services planning to this population.

\section{The Experience of People with Dementia and their Caregivers during Acute Hospitalization}

G. Lemay ${ }^{1}$, L. Garcia 1 , W. B. Dalziel ${ }^{1}$, L. McCleary ${ }^{2}$, N. Drummond ${ }^{3}$. ${ }^{1}$ University of Ottawa, Ottawa, ON; ${ }^{2}$ Brock University, St. Catharines, ON; ${ }^{3}$ University of Alberta, Edmonton, $\mathrm{AB}$.

Due to the growing number of individuals suffering from dementia, many will require acute hospital care as other indirectly related conditions appear throughout the course of the disorder. Significant concerns have been raised about the provision of quality care. Understanding their experiences is therefore essential. 
As part of a larger-scale study, semi-structured interviews were conducted with caregivers and people with dementia dyads after acute hospitalization. Data were analyzed using qualitative content analysis.

Twenty-nine participants including 25 family caregivers and 4 caregivers/people-with-dementia dyads experienced hospitalization and commented primarily on the negative experience. Despite the importance of caregiver involvement, and evidence of caregiver burden, they commented they were rarely included in care plans and lacked support. Continuity of care was said to be affected by absent communication amongst health-care team members. The acute hospital process and environment were perceived as not supportive of dementia person-centred care principles, with reports of poor staff knowledge and recognition of the disorder.

The three important themes from this study - the importance of caregivers, communication challenges, and how the processes/environment of the acute hospital is not an ideal place for a person with dementia - raise important issues about care provision. These findings are supported by prior research that states that the acute-care hospital is not adapted to care for people with dementia.

Reports of acute-care hospital experiences of people with dementia and their caregivers provide insights about gaps in care delivery. These findings can influence the development of further initiatives for care improvement.

\section{Development of the Clinical Evaluation Platform for CIMA-Q}

A. Morinville ${ }^{1}$, J.-M. Villalpando ${ }^{1}$, M.-J. Kergoat ${ }^{1}$, S. Gauthier $^{2}$, H. Chertkow ${ }^{2}$, C. Bocti ${ }^{3}$, L. Verret ${ }^{4}$. ${ }^{1}$ Université de Montréal, Montreal, QC; ${ }^{2}$ McGill University, Montreal, QC; ${ }^{3}$ Université de Sherbrooke, Sherbrooke, QC: ${ }^{4}$ Université Laval, Quebec, QC.

The Consortium pour l'identification précoce de la Maladie d'Alzheimer - Québec (CIMA-Q) aims for earlier diagnosis of Alzheimer's disease, before the dementia stage, and to better understand its causes, in order to develop effective therapies and identify at-risk populations. The clinical cohort group adapted or developed clinical assessment tools to evaluate both global and cognitive health status, as well as to identify health and lifestyle related risk factors, both at the start of the study and over time. This questionnaire had to be completed in under 2.5 hours, and needed to be useful to evaluate both healthy and cognitively impaired elderly individuals.

Longitudinal studies of aging and Alzheimer's were consulted as well as Cognition Clinics evaluation tools in order to determine themes that are most commonly assessed. Final selection was made by a team of experts (clinicians from memory clinics across Quebec). Within each topic, most commonly used/best questions and questionnaires were evaluated by the team of experts. When possible, a validated questionnaire was used, in order to make comparisons with other studies possible. Questions were created and adapted if a validated questionnaire could not be found.

Three different tools were created, both in French and in English: (1) a telephone screening interview (15 minutes), (2) a participant evaluation (2.5 hours), and (3) an informant questionnaire (15 minutes). All were piloted in a group of elderly volunteers. Fifteen validated questionnaires are included, such as the MoCA, a telephone MMSE, functional autonomy scales, and a depression scale.

The CIMA-Q clinical assessment tools can be used to evaluate both cognitively-impaired and non-impaired participants.

The inclusion of validated questionnaires should permit comparisons with previous and future longitudinal studies.

\section{Evidence Base for Yoga and Yoga Therapy with Geriatric Populations}

M. Pearson. International Association of Yoga Therapists, Little Rock, AR.

To provide an evidence base to support the use of safe and appropriate yoga instruction and yoga therapy for an aging population including geriatric and palliative care clients. This presentations will share the benefits of yoga postures, breath work, mindfulness, and meditation on the physiological, biochemical, psychological, and spiritual health and well-being of geriatric populations.

A review of the current research on the benefits of yoga therapy practices for addressing common physical diseases and the effects of sedentary lifestyles for aging populations including geriatric and palliative care patients will be presented.

- Specific recommendations for health concerns \& conditions such as: Alzheimer's, dementia, COPD (Chronic Obstructive Pulmonary Disease), cardiac rehab, anxiety, depression, cardiovascular disease, chronic lower respiratory disease, diabetes, cancer, injuries from accidents, joint pain, hearing loss, vision impairment

- Integrative care for palliative care patients

- Best practices for geriatric and palliative care patients

- Psychological issues

- $\quad$ Spirituality for aging

The implications of this research for integrating yoga, breathing exercises, laughter yoga, spirituality, mindfulness and meditation into existing geriatric healthcare programs. 
The current research provides a strong evidence base for incorporating yoga and yoga therapy into geriatric health and wellness programs to support the aging population in maintaining physical health, biochemical and psychological health, and well-being.

\section{Delirium: A Medical Emergency}

S. Tully, R. Sinyi, A. Carroll. University Health Network, Toronto, ON.

The literature indicates that delirium is increasing in incidence in the acute medically ill patient. The prevalence of delirium ranges from $6 \%$ to $56 \%$ in hospital settings and costs upwards of 17 million dollars/year. There are many risk factors known to contribute to delirium, resulting in the complexity of identifying, assessing, and managing a patient that develops delirium.

Hospital-acquired delirium is a concern for the geriatric population. The evidence states that the development of delirium influences a patient's hospital course, increasing length of stay, and is associated with poor long-term physical functional and cognitive outcomes. Moreover, psychological distress to family and care givers is significant.

Several studies have shown gaps in health-care professionals' knowledge, skills, and comfort regarding management of delirium, including inconsistent use of screening instruments and poor adherence to management protocols. The management can be complex, requiring the collective expertise of the interprofessional team.

To address delirium issues this poster was created to improve understanding of various types of delirium as well as to highlight prevention and management strategies.

Delirium was made a focus at UHN and the poster was developed to summarize UHN's initiatives. These included:

- Identifying risk factors and causes of delirium

- Discussing measures for delirium prevention

- Explaining assessment and management of patients with delirium

- Demonstrating the use of CAM assessment

- Discussing CAM assessment and interventions documentation

- Delirium Prevention and Management

- Results

- The Communication Strategy

- Tools and policies

- Education

The poster promoted awareness for staff about delirium and promoted the UHN initiative (see "Conclusions").

The literature indicates that $40 \%$ of delirium can be prevented. It is clear that interprofessional education regarding identification, prevention and management is paramount to reduce the risk of delirium development and associated long-term deleterious outcomes.

79. No Difference in Opioid Administration to Elderly Patients between Rural and Urban Emergency Departments in Ontario: Results from a Retrospective Cohort Study

N. Dattani ${ }^{1}$, N. Tassone ${ }^{1}$, D. Melady 1 , A. Costa ${ }^{2}$, W. K. Milne ${ }^{3}$. ${ }^{1}$ University of Toronto, Toronto, ON; ${ }^{2}$ McMaster University, Hamilton, ON; ${ }^{3}$ Western University, London, ON.

While most clinicians acknowledge that opioid analgesia can be an important component of ED pain management, it is well documented that ED opioid administration and prescribing is influenced by age, with older patients being less likely to receive opioids than younger patients.

Retrospective chart review involving three rural emergency departments and one urban emergency department studying a total of 92 patient visits at the rural sites and 193 patient visits at the urban site. Information describing the emergency department visit and administration of analgesia in the emergency department was abstracted.

$12 \%$ of rural patients and $32 \%$ of urban patients received opioid analgesia from the time of triage to discharge from the emergency department ( $p=.0003$ for comparison). However, after adjusting for possible confounders, rurality was not associated with a decreased likelihood of opioid administration (odds ratio, 0.722; 95\% CI, 0.291-1.791). 10\% of rural patients and $24 \%$ of urban patients received acetaminophen $(\mathrm{p}=.0038$ for comparison), while $14 \%$ of rural patients and $8 \%$ of urban patients received a non-steroidal anti-inflammatory drug (NSAID) ( $\mathrm{p}=.0918$ for comparison).

The overall rate of opioid administration was low in both settings. Interestingly, the rate of acetaminophen administration was even lower in both settings, and the rate of NSAID administration was higher than that of opioid administration in the rural settings combined. Clinical practice guidelines for treating pain in the elderly state that acetaminophen is generally considered to be the first-line pharmacological treatment, and that NSAIDs should be used cautiously due to their side effect profile.

Rurality did not influence emergency department administration of opioids to elderly patients in pain after adjusting for possible confounders.

80. Do We Do What Patients with Dementia and Their Caregivers Need? A Systematic Mixed Studies Review on Case Management in Primary Care

V. Khanassov, I. Vedel. McGill University, Montreal, QC. 
Patients with dementia and their caregivers express different needs such as coping with memory loss, information on the disease, emotional support. As a key intervention of the Alzheimer Plan of Quebec, case management (CM) has been implemented in Family Medicine Groups (FMGs) to address their needs. The purpose of this study is to identify the needs of the patient-caregiver dyad and if $\mathrm{CM}$ meets their expectations.

A systematic mixed studies review was conducted to integrate the results from studies with diverse designs (quantitative, qualitative, mixed methods studies) on needs of the dyad and CM, published in English or French up to 2014. CM comprises case finding, assessment, care planning, and monitoring and includes a family physican working with a case manager. Two reviewers selected title/abstract, full-texts, and appraised quality (using the Mixed Methods Appraisal Tool) independently. Synthesis (a sequential explanatory design): (i) development of the categories of needs based on the deductive approach; (ii) identification of the needs that $\mathrm{CM}$ targets to address; (iii) juxtaposition of the needs of the dyad to the needs that $\mathrm{CM}$ targets to improve.

54 studies were included; 8 studies concern CM. Four main categories of needs were identified: needs for information and knowledge, needs to maintain normality, caregiver needs, and access to care.

Overall CM effectively addresses the needs of the dyad living in the community. Impact of $\mathrm{CM}$ on access to care (e.g., access to trained family physicians) is not studied.

This study highlighted a knowledge gap on the impact of $\mathrm{CM}$ on access to care. These results will guide the conduit of experimental study on the needs of the dyad receiving care within CM implemented in FMGs of Quebec.

\section{Identifying Care Gaps in the Secondary Prevention of Fragility Fracture of the Hip}

M. von Maltzahn, S. A. Stewart, G. Johnston. University of Saskatchewan, Saskatoon, SK.

Patients who have sustained fragility fractures remain at high risk for future fracture without appropriate boneprotective interventions. The goal of our investigation was to examine the rate of bisphosphonate prescription within three months of discharge from hospital among patients with fragility fracture of the hip admitted to the Orthopedic Service at the Royal University Hospital, in Saskatoon. The data from this study will inform future interventions tailored to improve secondary prevention of fragility fractures in Saskatchewan.

A chart review of 114 patients over the age of fifty with a fracture of the hip was completed. Patients whose mechanism of injury was not consistent with fragility fracture were excluded, along with those receiving treatment with bisphosphonates at admission, or creatinine clearance less than $35 \mathrm{~mL} / \mathrm{min}$. Data were matched with the provincial Pharmaceutical Information Program (PIP) to assess for prescription of bone protective therapy within three months of discharge.

Five patients received prescriptions for bisphosphonate therapy (7\%, 95\% CI: $[1 \%, 13 \%])$, and 70 of the eligible patients did not receive treatment $(93 \%, 95 \%$ CI $[87 \%, 99 \%])$. A total of 23 patients were precluded from receiving bisphosphonate therapy, and an additional 16 patients presented with fragility fracture of the hip while on bisphosphonate treatment.

These findings reveal an identifiable care gap in the secondary prevention of fragility fracture of the hip in Saskatchewan. Patients who have sustained fragility fractures are at risk of adverse outcomes including loss of function and decreased quality of life.

Further study to improve the management of this cohort is under way. We have begun a knowledge translation intervention, supplementing discharge summaries with information to identify patients in need of treatment and further follow-up to prevent future fragility fracture.

\section{Development of Competencies for a Geriatric Ambulatory Care Subspecialty Program Using Consensus of Experts}

C. Tessier-Bussieres, S. Strau, B. Liu. University of Toronto, Toronto, ON.

Ambulatory care is a key component of geriatric medicine subspecialty training but currently there are no standardized core competencies in this domain. The goal of this project is to develop a set of competencies for geriatric ambulatory medicine that are essential for the geriatric subspecialty residents to master by the end of their curriculum to become independent in their professional practice, offer the best care possible, and respond to the increasing demand for the ambulatory care of older patients.

We are completing a multiphase project including an environmental scan, modified Delphi, and an in-person meeting with relevant experts to develop the list of core competencies.

In the first phase, we identified 151 core competencies from the current literature and lists provided by 7 geriatric program directors in Canada. They were divided into six domains (medical expert, manager, collaborator, communicator, scholar, and professional). The modified Delphi is under way and we expect 38 leaders in geriatric medicine to participate in the surveys.

At the time of the 2015 CGS Annual General Meeting, data from the Delphi will be available and competencies 
that should definitively be included or eliminated from the final list will be identified. Competencies with discordant results will be compiled and presented for discussion during the experts meeting.

The final goal of this project is to produce an exhaustive and concise list of competencies that cover the essential aspects of ambulatory care for elderly. We are hoping to create a valid and useful tool to develop ambulatory geriatrics training curricula and to emphasize skills specific to ambulatory medicine.

83. Experiences of Patient, Care Providers, and Professional Perspectives on Barriers and Facilitators to Quality Care in Advanced Heart Failure

\section{J. Li, N. Azad. University of Ottawa, Ottawa, ON.}

Elderly with advancing chronic heart failure (CHF) experience high levels of morbidity and mortality, similar to patients with common cancers. There is a gap in optimal quality of care and the real experiences for these patients. Even in the terminal stages, patients and caregivers are heavily and unnecessarily burdened by health-care services that are poorly coordinated and offer fragmented care. There is evidence that these poor experiences and the lack of understanding of their preferences could be improved to a large extent by simple organizational and process improvement rather than complex clinical mechanisms.

This study examines patient, carer, and professional perspectives on current management of advanced HF and barriers and facilitators to improved care.

Qualitative study involving semi-structured interviews and focus groups with advanced HF patients $(\mathrm{N}=20)$, carers $(\mathrm{N}=20)$, and professionals $(\mathrm{N}=30-40)$. Some questions are testing the knowledge, others attitude, opinion, and experience. The questionnaires for the one-on-one interviews are different from the focus group interviews. A conversational style of communication, starting the questionnaire with an introductory statement and a non-threatening and openended easy question to warm up is used.

The literature highlights that little progress is being made to improve care experiences for those with advanced HF. Even in the terminal stages, patients and caregivers are heavily and unnecessarily burdened by health-care services that are poorly coordinated and offer fragmented care.

There is evidence that these poor experiences could be improved to a large extent by simple organizational rather than complex clinical mechanisms.

We will discuss the result of this study and provide recommendations.

\section{Medical Trainees' Experiences with Complex Patients}

R. Joseph, L. Diachun, S. Cristancho, L. Lingard. Western University, London, ON.

Health care has entered an era of complexity. Patients often suffer from multiple comorbidities, and the growing cohort of elderly patients present with medical, psychiatric, functional, and social complexity. There is growing recognition that the ability to handle complex cases is a critical physician competency which should be developed in trainees. We aim to explore how medical trainees conceptualize clinical complexity, how well-prepared they feel to handle complexity, and how complex encounters influence residency selection.

In this qualitative study, to date, 13 participants (12 3rd-year students in their 11-12th month of clerkship and 1 4th-year elective student) engaged in a two-part interview process. First, students drew two rich pictures that represented complex clinical scenarios: one exciting, and one frustrating, overwhelming, or uncomfortable. Second, the pictures were used to guide a semi-structured interview. Interview transcripts were coded, compared, and analyzed using constructivist grounded theory principles.

Preliminary emerging categories for how trainees conceptualize complexity include role certainty, perceptions of learning, and belonging. These suggest the notion of active engagement as a key process influencing how clinical complexity is perceived; when actively engaged, students described the case as complex and exciting versus complex and frustrating. Active engagement may also influence residency selection as feelings on complexity and mentorship appear to be connected when describing specialty preferences.

Preliminary results suggest that the social process of learning, in relation to feelings of engagement, is a strong determinant of how medical trainees experience complex patients and the choices that result from these encounters.

Medical students must be trained to handle complex cases. Future direction for this study includes recruitment of additional participants, and further exploration on trainees' preparedness for clinical complexity and how the pre-clinical curriculum can better support the development of this competency.

\section{Podcasts in Career Planning for Geriatric Medicine: What Kind of a Doctor Do You Want to Be?}

M. Latrous ${ }^{1}$, A. M. Byszewski ${ }^{1}$, K. Bezzina ${ }^{1}, \mathrm{~A} . \mathrm{Vo}^{1}, \mathrm{R}$. Ellen ${ }^{2} .{ }^{1}$ University of Ottawa, Ottawa, ON; ${ }^{2}$ The Ottawa Hospital, Ottawa, ON. 
The recent Royal College of Physicians and Surgeons report highlighted areas of medicine that are over-serviced, but also emphasized those that are under-serviced, including geriatric medicine. The goal of this project was to develop a multimedia podcast template that would inform medical students about various career choices. Geriatric Medicine served as the prototype podcast.

A needs assessment was done by surveying existing resources (CaRMS, AAMC, AFMC, CFMS) and conducting an online survey of medical students. Based on these results, a list of interview questions was developed for this video podcast.

A Sony camcorder was used for recording interviews in Ottawa while Skype and ScreenFlow were used to interview and record physicians and residents nation-wide.

Interviews were conducted with physicians and residents working in Geriatrics. Relevant links and resources were added to the podcast. It is now housed on the CGS website and the TOH Department of Medicine webpage. As well, the CFMS and AAMC plan to add it to their websites. The podcast was evaluated by medical students via a questionnaire.

Using a podcast framework to educate trainees on career choices is a novel idea. The results from this project may be used as a template for other postgraduate training programs and this podcast may be used to raise awareness of Geriatrics as a career choice.

Podcasts are a novel multimedia approach for demonstrating career opportunities to medical students, when considering residency training options.

\section{Delirium Screening of Older Adults at Mount Sinai Hospital (MSH): Compliance with Screening and Barriers and Needs Assessment}

A. Canfield ${ }^{1}$, C. Turchet ${ }^{1}$, L. Dan ${ }^{1}$, C. Fan-Lun ${ }^{2}$, L. Mantas $^{2}$, S. Sinha ${ }^{2}$, L. Burry 2 , N. Tabbara ${ }^{3} .{ }^{1}$ University of Toronto, Toronto, ON; ${ }^{2}$ Mount Sinai Hospital, Toronto, ON; ${ }^{3}$ University of Waterloo, Waterloo, ON.

Delirium is an acute confusional state that is most commonly seen in older adults and is a predictor of poor outcomes. Despite being a quality of care indicator, evidence shows that delirium is often unrecognized. This limits the possibility of timely management. The objectives of this study are to determine the rates of delirium screening at MSH and to identify barriers and the needs of health-care professionals to better facilitate daily delirium screening.

We conducted a retrospective chart review of adults 65 years or older admitted to one of four units - Acute Care for the Elderly, ICU, general medicine unit, and orthopedic surgery - between September 2010 and October 2013. The survey consists of 4 sections: Demographics, Delirium,
Delirium Screening, and Education \& Resources, to be administered to health-care professionals at MSH that screen for delirium in the above-mentioned study subjects. The survey was developed in 4 phases: Item Generation, Item Reduction, Question Formatting, and Validation.

At the time of abstract writing (465 charts), the rate of total compliance with daily delirium screening was $60 \%$, the rate of screening within 24 hours of hospital admission was $62 \%$, and the rate of hospital-acquired delirium was $19 \%$. The survey was in the validation phase and to be distributed to staff in March 2015.

We hypothesize that many of the perceived barriers identified will be possible to target through the availability of resources, and that identifying the needs of health-care professionals will allow future resources to be appropriately chosen to enable improved screening rates.

This approach will allow more cases of delirium to be identified and opportunely managed at MSH to reduce negative outcomes.

\section{The Effects of Living Status on Cognitively Impaired Older Adults}

L. Rodrigues, K. Gopaul, A. Islam, M. Montero-Odasso. Parkwood Institute, London, ON.

In older adults with Mild Cognitive Impairment (MCI), a precursor to dementia, deficits in cognition can have negative impacts on well-being and quality of life. Research has shown depression increases risk of cognitive decline. Among the elderly, those who live alone are at risk of developing depressive symptoms. There is a lack of information from relevant literature on whether individuals with $\mathrm{MCI}$ are at risk for further cognitive deterioration depending on their relationships (marriage and/or companionship) and living arrangement (alone/with a partner).

This study is to investigate the effect of relationship status and living arrangement on memory and cognition, and whether depression mediates these effects in the elderly population diagnosed with MCI.

Data from the ongoing "Gait and Brain Study" cohort will be used to assess forty older adults with MCI who live alone, and forty with MCI who live with a partner. The Mini Mental State Examination (MMSE) will be used to assess cognition and depressive symptoms will be assessed using the Geriatric Depression Scale (GDS) every six months over a 2-year period.

This study is in its preliminary stages; results are pending.

We expect that individuals who live alone, in comparison to individuals living with a partner, will demonstrate greater declines in cognitive function, as evidenced by lower MMSE scores, and will experience greater depressive symptoms, represented by higher GDS scores. 
There is currently a lack of understanding whether relationship status and living arrangement, mediated by depression, affect cognitive function in individuals with MCI. This study may provide insight into possible social and psychological risk factors, further contributing to cognitive decline, that need to be addressed in the care of older adults with MCI.

88. A Needs Analysis Questionnaire to Improve the Coordination of Health Care and Services Delivered to Seniors with Multiple Chronic Diseases in the RUIS McGill

\section{H. Zhao, A. A. Tanon. McGill University, Montreal, QC.}

The McGill Centre of Excellence on Aging and Chronic Diseases (CEViMaC) aims to design a needs analysis questionnaire that can measure the performance gaps between the current situation and an ideal situation in terms of coordination of health care and services for seniors with multiple chronic diseases in the McGill Réseau Universitaire Intégré de Santé (RUIS McGill). This research project represents an important procedure as it will guide the CEViMaC in providing appropriate clinical support to health organizations within RUIS McGill.

The Kaufman methodology of needs analysis at the strategic level and a conceptual framework of health-care coordination were selected to guide the questionnaire's design process. A scoping review was performed to identify strategic plans of relevant health-care organizations: pertinent goals of coordination were analyzed and converted into items for the questionnaire. The measurement scale and the basic visual design of the questionnaire were based from similar research studies. Finally, qualitative interviews with a sample of healthcare professionals were conducted to test the questionnaire.

According to the interviewees, the visual design and the measurement scale were intuitive, and the items reflected broadly their professional concerns. However, some instructions and questions lacked coherence and contained inaccurate terminology. Additionally, the completion of the questions required a considerable amount of time.

Reformulating the instructions and the questions, and arranging thematically the sequence of questions, are necessary to facilitate comprehension. Otherwise, the number of questions should be reduced to ensure a favorable response rate.

A team of suitable experts, further interviews, and adjustments are needed to improve the measurement tool in addressing adequately the dimensions of care and service coordination. Use of incentives should be explored to boost response rate upon the questionnaire's official launch.

\section{Does Low Life Satisfaction Predict Dementia over a Five-Year Period in Cognitively Intact Older Adults?}

\author{
L. Peitsch, S. L. Tyas, V. Menec, P. St. John. University of \\ Manitoba, Winnipeg, MB.
}

Low life satisfaction (LS) predicts poor outcomes and may predict dementia. We sought to determine if LS predicts dementia over a five-year period in older adults with normal cognition at baseline.

Secondary analysis of a population-based cohort study of 1,751 older adults, with initial assessment in 1991 and follow-up five years later; 1,028 were cognitively intact at time 1 , and alive and had complete data at time 2 when 96 were diagnosed with dementia. Measures were age, gender, and education, which were self-reported. LS was assessed using the Terrible-Delightful scale, which measures overall LS and aspects of LS (e.g., health, finances, friendships) using separate 7-point scales. Cognition was assessed using the modified Mini-mental State Examination (3MS) and a clinical exam for those scoring $<78$. Cognitive status was categorized as intact, dementia, or Cognitive Impairment, No Dementia (CIND). Analyses were adjusted for age, gender and education.

There were few cognitively intact people at time 1 with low LS. Overall LS predicted death, dementia, and CIND five years later. Those with low overall LS were more likely to die than to develop dementia. In logistic regression models, the unadjusted Odds Ratio (OR; 95\% confidence interval) for dementia at time 2 was $0.72(0.55,0.95)$ per point on the LS scale, with the adjusted OR $0.68(0.50,0.93)$. However, no individual domain of LS predicted dementia, although the competing risk from mortality was high for some items.

The risk of death exceed the risk of dementia over the five-year interval.

A basic global measure of LS predicts dementia over a five-year period in older adults without cognitive impairment. No individual aspect of LS predicted dementia.

\section{Can Balance and Cognition Help to Identify At-Risk Individuals?}

Florence St-Onge ${ }^{1}$, Elisabeth Charlebois ${ }^{1}$, Anil Nigam ${ }^{1}$, Louis Bherer ${ }^{2}$, Sarah Fraser ${ }^{3}$. ${ }^{1}$ University of Montreal, Montreal, QC; ${ }^{2}$ Concordia University, Montreal, QC; ${ }^{3}$ McGill University, Montreal, QC.

Cardiovascular disease, related risk factors, and falls are all well-known predictors of cognitive decline in older adults. In a longitudinal study assessing cardiovascular health, cognition, balance, and dual-task walking, we evaluated baseline differences in balance outcomes and neuropsychological assessments to see if poor balance and 
poor cognition were associated with individuals who have poor cardiovascular health.

The Cardiac group ( $\mathrm{CG}, \mathrm{N}=23)$ included individuals with $\geq$ two cardiovascular risk factors and coronary patients. Healthy group ( $\mathrm{HG}, \mathrm{N}=16)$ included individuals with < two risk factors.

Mean age: HG 64.7 years old SD 4.3 years, CG 69.9 years old SD 4.9 years.

The Montreal Cognitive Assessment (MoCA) was measured prior to balance assessment. Ten-second balance assessment were performed using a Matscan platform and included two eyes-open conditions (bipedal and unipedal).

Initial comparisons of center of pressure measures between groups revealed significant differences in several measures (mean velocity [MV], mediolateral velocity $[M L V])$. After controlling for age and sex differences between groups, a mixed ANOVA (group by balance condition) revealed significant interactions in $\mathrm{MV}$ and MLV, such that CG performed more poorly than HG in the unipedal conditions (MV: $\mathrm{p}=.04$, MLV: $\mathrm{p}=.02$ ) with no significant differences in the bipedal conditions (MV: $\mathrm{p}=$ .86 , MLV: $\mathrm{p}=.628)$. MLV also negatively correlated with MoCA scores in the whole sample $(\mathrm{p}=.05)$.

This study suggests that $C G$ have poorer balance control than HG. Simple tests with a balance platform reveal differences that identify at risk groups and relate to lower MOCA scores.

Early assessments of balance status can help to identify older individuals at risk of cognitive decline, allowing early intervention to prevent falls and further cognitive decline.

\section{Mild Cognitive Impairment: Cognitive Testing and EEG Changes}

F. Knoefel ${ }^{1}$, M. Breau ${ }^{1}$, L. Sweet ${ }^{1}$, C. Lord $^{1}$, R. L. Zunini ${ }^{2}$, V. Taler ${ }^{2}$, B. Wallace ${ }^{3}$, R. Goubran ${ }^{3} .{ }^{1}$ Bruyère Continuing Care, Ottawa, ON; ${ }^{2}$ University of Ottawa, Ottawa, ON; ${ }^{3}$ Carleton University, Ottawa, ON.

Speed of information processing can be affected in Mild Cognitive Impairment (MCI) and may play a role in activities such as driving. Electroencephalography (EEG) may have a role in assessing cognition, because it measures current changes to the level of milliseconds. In cognitive testing, the EEG signal is averaged to yield a waveform termed an "event-related potential" (ERP). This study compares the results of traditional cognitive testing and ERP n-back testing in patients with MCI and healthy controls (HC).

Thirteen MCI patients were recruited from the Bruyère Memory Program, and $9 \mathrm{HCs}$ were recruited from the general population. Cognition was tested using the MoCA, RBANS, and Trails A \& B. EEGs were measured using
NeuroScan NuAmps 4.3 and analysed using Brain Analyzer 2.0. Participants performed n-back cognitive tests to elicit working memory relevant ERPs.

There were significant differences between clinical test results of MCI and $\mathrm{HC}$ groups; e.g., mean Trails B time for MCI was 173.5 seconds compared to 76.4 seconds in the HC $(\mathrm{p}<.001)$. In addition, the HC group had more correct responses and responded more quickly than the MCI patients in all $3 \mathrm{n}$-back conditions $(\mathrm{p}<.003)$. There was also a significant delay in the P200 component at midline and left hemisphere centro-posterior electrodes in MCI patients when compared to HC $(\mathrm{p}<.04)$.

This MCI group performed less well on traditional cognitive testing, was slower, and made more mistakes in the n-back testing, and showed a significant delay in the P200 component compared to healthy older controls.

Further work will be required to clarify the role of ERP in measuring decreases in speed of processing in older adults with cognitive impairment.

\section{Longitudinal Mobility Monitoring: Preliminary Results}

F. Knoefel ${ }^{1}$, T. Grant ${ }^{1}$, Z. Ren ${ }^{2}$, R. Goubran ${ }^{2}$, M. Bilodeau ${ }^{3}$, H. Sveistrup ${ }^{3}$, J. Jutai ${ }^{3}$. ${ }^{1}$ Bruyère Continuing Care, Ottawa, $\mathrm{ON} ;{ }^{2}$ Carleton University, Ottawa, ON; ${ }^{3}$ University of Ottawa, Ottawa, ON.

The current practice to monitor mobility is periodic clinical assessment. Technology is now available that would allow continuous monitoring. The purpose of this study was to track mobility changes in 25 community-dwelling, frail older adults over one year, with the ultimate goal being the early detection of mobility decline.

Clinical measures, including Timed Up \& Go (TUG) and gait speed, were taken monthly for frail older adults living in the community. Under-mattress pressure-sensitive mat (S4 Sensors Inc.) data were collected continuously over the same time period and analyzed with custom-designed software.

Among the first 15 participants, data were collected over an average period of 10 months. Of these participants, there were 8 that showed a clinically meaningful decline, 3 that showed a meaningful improvement and 4 that showed stability in mobility using the clinical measures.

Data from an 85-year-old male, with a history of cerebrovascular accidents, will be presented. Over the period June 2013 to May 2014, he went from using a cane to requiring a wheeled walker, his gait speed dropped from 0.70 to $0.47 \mathrm{~m} / \mathrm{s}$ and his TUG increased from 23.9 to $37.7 \mathrm{~s}$. This will be compared to changes in sum of pressures and centre of pressure velocity magnitude data extracted from the mat. 
Distinct changes in data analyzed by the pressuresensitive mat were associated with functional decline in the case presented. Further data analyses are ongoing to determine whether continuous monitoring of bed mobility can facilitate the early detection of mobility decline.

To our knowledge, this is the first time that bed transfer data has been measured continuously over a year in frail, community-dwelling older adults.

\section{Towards Consensus on Quality Assurance Indicators for Primary Care Memory Clinics}

G. Heckman, V. Boscart, L. Hillier, L. Lee, F. Molnar, D. Seitz, P. Stolee.

Introduction: Increasing numbers of primary care memory clinics (PCMCs) are being established in Ontario to provide much-needed system capacity for dementia care and efficient integration with specialty services. There is a need for routine integration of standardized quality indicators (QI) into PCMC care processes to ensure high-quality care. The purpose of this project was to develop consensus on the most relevant QIs for PCMCs to promote quality care and program fidelity and sustainability.
Methods: A Delphi survey of PCMC clinicians and specialists was used to develop consensus on QIs for use in PCMCs. A list of QIs was identified through a systematic literature review. Survey participants were asked to rate the QIs according to their importance and feasibility in PCMCs. Mean ratings determined relative rankings of importance. Two survey rounds were conducted involving 179 and 88 participants, respectively. QIs ranked below a pre-specified threshold or not readily measurable in a primary care setting were eliminated.

Results: Consensus was reached on 18 QIs. These included criteria for referrals to specialists; assessment, reassessment, and documentation requirements for diagnoses of mild cognitive impairment and dementia; criteria for diagnostic testing; medication reviews; discussing the risks and benefits of cholinesterase inhibitors and of antipsychotics; use of stroke prophylaxis in the presence of vascular risk factors; assessment for safety risks; counselling on driving risks and fitness to drive assessment; and advanced care planning, including identification of substitute-decision makers.

Conclusions: A set of QIs for PCMCs has been identified. These indicators will form the basis of a quality assurance framework to enhance primary and collaborative care and that can be used in further studies to evaluate the quality of dementia care models in primary care.

\section{CANADIAN CONSORTIUM ON NEURODEGENERATION IN AGING (CCNA) ABSTRACTS}

\section{CCNA 1. New Insight Into How Vitamin D Influences Mobility and Fall Risk in Seniors: A Magnetic Resonance Spectroscopy of the Caudal Primary Motor Cortex}

C. Annweiler ${ }^{1}$, R. Bartha ${ }^{1}$, V. Hachinski ${ }^{1}$, M. MonteroOdasso $^{1}$, O. Beauchet ${ }^{2} .{ }^{1}$ University of Western Ontario, London, ON; ${ }^{2}$ Angers University Hospital, Angers, France.

Vitamin D is involved in brain physiology and lowerextremity function. We investigated spectroscopy in a cohort of older adults to explore the hypothesis that lower vitamin D status was associated with impaired neuronal function in caudal primary motor cortex (cPMC) measured by proton magnetic resonance spectroscopic imaging. Twenty Caucasian community-dwellers (mean \pm standard deviation, $74.6 \pm 6.2$ years; $35.0 \%$ female) from the "Gait and Brain Study" were included in this analysis. Ratio of $\mathrm{N}$-acetyl-aspartate to creatine (NAA/Cr), a marker of neuronal function, was calculated in cPMC.

Participants were categorized according to mean NAA/Cr. Lower vitamin D status was defined as serum 25-hydroxyvitamin D (25OHD) concentration $<75$ nmol/L. Age, gender, number of comorbidities, vascular risk, cognition, gait performance, vitamin D supplements, undernourishment, cPMC thickness, white matter hyperintensities grade, serum parathyroid hormone concentration, and season of evaluation were used as potential confounders.

Compared to participants with high NAA/Cr $(\mathrm{N}$ $=11$ ), those with low NAA/Cr (i.e., reduced neuronal function) had lower serum 25OHD concentration ( $\mathrm{p}=$ .044 ) and more frequently lower vitamin D status ( $p$ $=.038$ ). Lower vitamin $\mathrm{D}$ status was cross-sectionally associated with a decrease in NAA/Cr after adjustment for clinical characteristics (beta $=-0.41, \mathrm{p}=.047$ ), neuroimaging measures (beta $=-0.47, \mathrm{p}=.032$ ) and serum measures (beta $=-0.45, p=.046$ ). Lower vitamin $\mathrm{D}$ status was associated with reduced neuronal function in $\mathrm{cPMC}$.

These novel findings need to be replicated in larger and preferably longitudinal cohorts. They contribute to explain the pathophysiology of gait disorders in older adults with lower vitamin D status, and provide a scientific base for vitamin D replacement trials. 
CCNA 2. Walking While Thinking: Neural Contributions to Dual-task Treadmill Walking in Younger and Older Adults

S. Fraser ${ }^{1}$, P. Pouliot ${ }^{2}$, F. Lesage 2 , O. Dupuy ${ }^{3}$, P. Roy ${ }^{4}$, L. Lehr ${ }^{4}$, L. Bherer ${ }^{5} .{ }^{1}$ McGill University, Montreal, QC; ${ }^{2}$ École Polytechnique de Montréal, Montreal, QC; ${ }^{3}$ Université de Poitiers, Poitiers, France; ${ }^{4}$ Université du Québec à Montréal, Montreal, QC; ${ }^{5}$ Center-Concordia University, Montreal, QC.

Everyday situations in which attention is divided (e.g., walking and thinking) can put older adults at risk for accidents and falls. In order to prevent falls and provide early interventions for cognitive-motor declines, additional research targeting neural contributions during dual-task walking is needed.This study assessed behavioural and neural changes during dual-task walking. Younger (YA; N $=19)$ and older adults $(\mathrm{OA} ; \mathrm{N}=14)$ walked on a treadmill at a self-selected pace while performing an n-back task with two difficulty levels (1- \& 2-back). Using a blocked design, changes in oxy- (HbO) and deoxy-hemoglobin (HbR) during single (ST: walk) and dual task (DT: walk $+n-b a c k)$ were acquired from 28 channels placed on the prefrontal cortex (PFC). Within each group and channel, a task by difficulty ANOVA was conducted.

For $\mathrm{HbO}$, a significant task effect $(\mathrm{ST}<\mathrm{DT})$ was found in 5 channels in YA and 7 channels in OA ( $p$ values $<.028)$. For HbR, task effects (ST $<\mathrm{DT})$ were found in $17 / 28$ channels for the YA and 24/28 channels for the OA ( $p$ values < .045). In four channels (left hemisphere), a significant task by difficulty interaction was found in the YA, in which the HbR concentration change in the 2-back DT was lower than the change in the 1-back DT, a finding not present in the OA. Both groups demonstrate changes in cerebral oxygenation during dual-task walking, with OA demonstrating these effects in more channels than YA. YA may have benefited from dual-task exposure as they have less activity in the harder dual-task condition.

These findings help clarify neural contributions to dual-task walking and can be used to inform intervention work on falls and cognitive-motor declines in aging.

CCNA 3. Pre-Admission Residence Impacts Magnitude of Functional Gains Achieved During Inpatient Rehabilitation among Older Adult with Dementia

S. Hunter, J. Wells, M. Montero-Odasso, G. L. Fat, R. Mackenzie. University of Western Ontario, London, ON.

While research has demonstrated cognitive impairment is a risk factor for functional decline and may affect rehabilitation compared to the cognitively intact, the characteristics that lead to rehabilitation success in people with dementia has not been evaluated. Our objective was to determine factors associated with functional gains among older adults with dementia during inpatient geriatric rehabilitation.

Retrospective cohort study: Consecutive subjects admitted to an inpatient geriatric rehabilitation unit with a dementia diagnosis $(\mathrm{N}=175$, age $83.1 \pm 7.2 \mathrm{y}, 55.4 \%$ female $)$ had mobility, cognitive and demographic data collected at admission and discharge. The Functional Independence Measure motor function (FIM-motor) subscale was used to estimate level of mobility. Gain in motor function was the difference between FIM-motor scores at admission and discharge. Multivariable linear regression evaluated the association between cognitive and demographic factors on mobility gains.

Functional gains were smaller with increasing care among pre-admission residence settings. The mean gain in mobility was $48.9 \%$ for "home without services," $44.6 \%$ for "home with services," $38.0 \%$ for "assisted living," and $-4.9 \%$ for "residential care." In regression analysis, compared to "home without services," average FIM-motor gains were lower by 4 points for "home with services" ( $p=.042)$, 5.6 points for "assisted living" $(p=.029)$ and 23.2 points for "residential care" $(p<.001)$. No other demographic or cognitive factors were associated with mobility gains. Only pre-admission place of residence was associated with mobility gains. More research is needed on the link between pre-admission function and loss of function on rehabilitation gains to refine positive prognostic factors associated with residence setting.

Age, MMSE, gender, comorbidities, and mobility aid use were not associated with mobility gains and should not be absolute contraindications for access to inpatient rehabilitation.

\section{CCNA 4. Mild Cognitive Impairment: Cognitive Testing and EEG Changes}

F. Knoefel ${ }^{1}$, M. Breau ${ }^{1}$, L. Sweet ${ }^{1}$, C. Lord ${ }^{1}$, R. Lopez Zunini $^{2}$, V. Taler ${ }^{2}$, B. Wallace ${ }^{3}$, R. Goubran ${ }^{3}$. ${ }^{1}$ Bruyère Research Institute, Ottawa, ON; ${ }^{2}$ University of Ottawa, Ottawa, ON; ${ }^{3}$ Carleton University, Ottawa, ON.

Speed of information processing can be affected in Mild Cognitive Impairment (MCI) and may play a role in activities such as driving. Electroencephalography (EEG) may have a role in assessing cognition, because it measures current changes to the level of milliseconds. In cognitive testing, the EEG signal is averaged to yield a waveform termed an "event-related potential" (ERP). This study compares the results of traditional cognitive testing and ERP n-back testing in patients with MCI and healthy controls (HC). Thirteen MCI patients were recruited from 
the Bruyère Memory Program, and 9 HCs were recruited from the general population. Cognition was tested using the MoCA, RBANS, and Trails A \& B. EEGs were measured using NeuroScan NuAmps 4.3 and analysed using Brain Analyzer 2.0. Participants performed n-back cognitive tests to elicit working memory relevant ERPs.

There were significant differences between clinical test results of MCI and $\mathrm{HC}$ groups, e.g., mean Trails B time for MCI was 173.5 seconds compared to 76.4 seconds in the $\mathrm{HC}(p<.001)$. In addition, the HC group had more correct responses and responded more quickly than the MCI patients in all $3 \mathrm{n}$-back conditions $(p<.003)$. There was also a significant delay in the P200 component at midline and left hemisphere centro-posterior electrodes in MCI patients when compared to $\mathrm{HC}(p<.04)$.This MCI group performed less well on traditional cognitive testing, was slower and made more mistakes in the n-back testing, and showed a significant delay in the P200 component compared to healthy older controls.

Further work will be required to clarify the role of ERP in measuring decreases in speed of processing in older adults with cognitive impairment.

CCNA 5. Age-related Hearing Loss and Gait Adaptations: Early Evidence for Postural Prioritization in the Elderly

V. Nieborowska ${ }^{1}$, K. Z. H. Li ${ }^{1}$, S.-T. Lau ${ }^{2}$, A. Novak ${ }^{3}$, J. Campos ${ }^{3}$, M. K. Pichora-Fuller ${ }^{4}{ }^{1}$ Concordia University, Montreal, QC; ${ }^{2}$ Wilfrid Laurier University, Waterloo, ON; ${ }^{3}$ Toronto Rehabilitation Institute, Toronto, ON; ${ }^{4}$ University of Toronto, Toronto, ON.

Age-normative hearing loss is linked with reduced cognitive functioning, mobility decline, and increased falls risk in older adults. This study examined age-related differences in allocation of cognitive resources between concurrent walking and listening tasks. Seventeen younger and twelve older adults with normal hearing participated. Three sentences (1 target, 2 maskers) were played simultaneously from different locations (left, center, right) in a virtual-reality street crossing scene. Target location probability $(100 \%$ versus $<75 \%)$ was varied. Participants reported the number and colour in the target sentences. Gait during self-paced treadmill walking was assessed with motion capture system using active markers positioned on the head, sternum, sacrum, and feet. There were 3 conditions: walking, listening, and walking while listening. Key dependent measures were listening accuracy, head and trunk position, and stride time variability.

Word recognition accuracy was significantly worse in (a) older than younger adults, (b) dual- than single-task, and (c) less predictable location probability. Preliminary kinematic analysis suggests increased peak trunk pitch, head pitch, and peak head roll rotation in older adults compared to younger adults. Older adults showed decreased head roll, peak head roll, and peak trunk pitch rotation from single-task walking to dual-task conditions but there was no such decrease in younger adults. Stride time variability (coefficient of variation) was greater in older adults under dual-task conditions than single-task walking while younger adults showed no trend. Listening performance in old age is particularly hampered when concurrently walking and when the signal location becomes less predictable. Furthermore, older adults' gait variability worsened when simultaneously listening.

The findings suggest that listening challenges affect mobility and provide early evidence to explain the link between age-related hearing loss and gait adaptations.

CCNA 6. Gait Performance and Mild Traumatic Brain Injury: A Pilot Study on the Effects of a Weighted Compression Vest

\section{A. BiancaPopa. Western University, London, ON.}

After a mild traumatic brain injury (mTBI), individuals frequently experience balance problems associated with sensory processing disorders. Pediatric research has shown that compression vests improved anxiety in children with the disorder. However, a significant gap exists in literature regarding the application of compression vests in adult injury populations. The purpose of this study is to measure whether the vest improves gait and if it can improve mobility and aspects of cognition.Twenty-eight mTBI participants will complete the Rivermead post-concussion symptoms questionnaire (measure of anxiety) and cognitive testing, acting as their own controls performing a series of walking tests under four dual-task conditions. The dual-task conditions will consist of walking while performing a cognitive task with and without the compression vest. Cognitive testing includes the MoCA, to assess global cognition, and Trail Making to assess executive function (EF). Gait variability (GV) will be collected as a measure of gait performance. Participants will be wearing over-sized shirts to keep assessors blind to weighted vest condition. There will be a one week wash-out period.

We expect that gait performance, specifically GV, under-dual task conditions will improve using the weighted compression vest - a mechanism that can be related to reducing anxiety and improving EF. This study may provide evidence that weighted compression vests can improve patients' gait performance, allowing them to better navigate in daily environments. If gait improves using the compression vest, it may suggest that GV is sensitive to brain function. 
This study may contribute to recommending the use of compression vests for older adults to improve gait performance.

CCNA 7. Understanding the Mechanism Underlying the Relationship Between Executive and Gait Functions Using Regional Cerebral Glucose Metabolism in Healthy Older Women

R. Sakurai ${ }^{1}$, K. Ishii ${ }^{2}$, Y. Fujiwara ${ }^{2}$, M. Yasunaga ${ }^{2}$, M. Montero-Odasso ${ }^{3}$. ${ }^{1}$ University of Western Ontario, London, ON; ${ }^{2}$ Tokyo Metropolitan Institute of Gerontology, Tokyo, Japan; ${ }^{3}$ Parkwood Institute, London, ON.

Low executive function is associated with gait dysfunction in older adults. However, little is known about the neural basis underlying this association. We aim to investigate the relationship between regional cerebral metabolic rates of glucose (rCMRglc) and executive / gait functions in healthy community-dwelling older women. One-hundred and forty-five older women (mean age [SD], 69.6 [6.6] years) who have normal global cognitive function (MMSE $>$ 26) underwent positron emission tomography using [F18]fluorodeoxyglucose (FDG-PET) to assess brain activity at rest. Within 6 months before and after the FDG-PET, TMT-A, TMT-B and maximum gait indices (speed, step length, and cadence) were measured.

The difference in time between TMT-A and TMT-B was calculated for a measure of executive function $(\triangle \mathrm{TMT})$. Associations between variables were examined using multiple linear regression analyses adjusted for demographic variables and comorbidity.

Lower $\triangle \mathrm{TMT}$ was associated with poor performance in maximum gait speed and cadence, independent of covariates. For the cerebral glucose metabolism, both lower $\triangle \mathrm{TMT}$ and lower maximum gait indices (speed and cadence) were associated with lower rCMRglc in the posterior cingulate and the primary sensorimotor cortices.The present study in healthy older adults suggests that the known relationship between low executive function and gait dysfunction in seniors may be explained by reduced metabolic activity in the posterior cingulate and the primary sensorimotor cortices. Assessing the neural activity in these brain areas may be helpful for identifying older adults at higher risk of experiencing mobility disability, falls and progressive MCI or dementia.

The posterior cingulate and the primary sensorimotor cortex may play important roles in both executive and gait controls.

CCNA 8. Effects of Exercise on Cognition in Parkinson's Disease: The Link Between Executive Functions and Gait
C. Silveira, B. Intzandt, Q. Almeida. Wilfrid Laurier University, Waterloo, ON.

In Parkinson's disease (PD), deficits in executive functions (EF) have been linked to impaired gait. Current research has suggested that exercise may be effective to treat cognitive deficits in PD. However, it remains unknown how changes in cognition might influence gait in PD. This study aimed to investigate the effects of an aerobic and a Parkinson'sspecific goal-based exercise on EF and gait in PD and whether changes in cognition and gait as a result of exercise are associated or independent. Forty-eight PD participants were randomized into an aerobic $(\mathrm{N}=23)$ or a goal-based $(\mathrm{N}=25)$ exercise group and attended 1-hour sessions $3 \mathrm{x} /$ week for 12 weeks. EF (Digit Span, Stroop Test, and Trail Making Test) and gait (single and dual tasks) were assessed pre and post exercise.

A group by time interaction for the Stroop interference condition approached significance $(p=.056)$, revealing that only the aerobic group performed better in this condition at post-test. In addition, all participants performed the Trail's part A faster after exercise $(p=.009)$. An interaction between time, walking task, and group for step time variability neared significance $(p=.058)$, demonstrating that, while goal-based exercise decreased variability in the singletask, aerobic exercise decreased variability in the dual-task condition at post-test. Participants decreased step time, increased cadence and step width after exercise, only in the dual-task condition $(p<.05)$. Negative correlations showed that more points in the digit span forward were associated with reduced step time (aerobic: $r=-0.41$; goal-based: $r=$ -0.48) and double support (aerobic: $r=-0.50$ ) variability during dual-task. This is the first study to demonstrate that exercise-related changes in EF may influence gait in PD.

This influence occurs primarily during dual-task and is dependent on the measure of EF.

\section{CCNA 9. Can Balance and Cognition Help to Identify At-Risk Individuals?}

F. St-Onge ${ }^{1}$, A. Nigam ${ }^{1}$, É. Charlebois ${ }^{2}$, L. Bherer ${ }^{2}$, S. A. Fraser $^{2}$. ${ }^{1}$ University of Montreal, Montreal, QC; ${ }^{2}$ Institut Universitaire de Gériatrie de Montréal, Montreal, QC

Cardiovascular disease, related risk factors, and falls are all well-known predictors of cognitive decline in older adults. In a longitudinal study assessing cardiovascular health, cognition, balance, and dual-task walking, we evaluated baseline differences in balance outcomes and neuropsychological assessments to see if poor balance and poor cognition was associated with individuals who have poor cardiovascular health. The Cardiac group $(\mathrm{CG}, \mathrm{N}=23$ ) included individuals with $\geq$ two cardiovascular risk factors 
and coronary patients. The Healthy group $(\mathrm{HG}, \mathrm{N}=16)$ included individuals with $<$ two risk factors.

Mean age : HG 64.7 years old SD 4.3 years, CG 69.9 years old SD 4.9 years.

The Montreal Cognitive Assessment (MoCA) was measured prior to balance assessment. Ten-second balance assessments were performed using a Matscan platform and included two eyes-open conditions (bipedal and unipedal).

Initial comparisons of center of pressure measures between groups revealed significant differences in several measures (mean velocity [MV], mediolateral velocity $[\mathrm{MLV}])$. After controlling for age and sex differences between groups, a mixed ANOVA (group by balance condition) revealed significant interactions in $\mathrm{MV}$ and MLV, such that CG performed more poorly than HG in the unipedal conditions (MV: $p=.04, \mathrm{MLV}: p=.02$ ) with no significant differences in the bipedal conditions (MV: $p=$ .86 , MLV: $p=.628)$. MLV also negatively correlated with MoCA scores in the whole sample $(p=.05)$. This study suggests that $\mathrm{CG}$ have poorer balance control than HG. Simple tests with a balance platform reveal differences that identify at-risk groups and relate to lower MOCA scores.

Early assessments of balance status can help to identify older individuals at risk of cognitive decline, allowing early intervention to prevent falls and further cognitive decline.

\section{CCNA 10. Benefits of Physical Exercise and Cognitive Training on Dual-Task Performance}

T. T. M. Vu ${ }^{1}$, M. Lussier ${ }^{1}$, L. Desjardins-Crepeau ${ }^{1}$, S. Fraser ${ }^{1}$, N. Berryman ${ }^{1}$, L. Bosquet ${ }^{1}$, D. Predovan ${ }^{1}$, M.-J. Kergoat ${ }^{1}$, K. Z. H. Li ${ }^{2}$, K. Vadaga ${ }^{2}$, L. Bherer ${ }^{2} .{ }^{1}$ Centre de Recherche de
l'Institut Universitaire de Gériatrie de Montréal, Montreal, QC; ${ }^{2}$ Concordia University, Montreal, QC.

Several studies have reported benefits of exercise interventions and cognitive training on cognitive performance in older adults, but the effect of combining both interventions has rarely been studied. 136 healthy older adults were randomly assigned to one of the 4 combinations: 1) aerobic training and dual-task training; 2) aerobic training and placebo computer; 3) stretching training and dual-task training; 4) stretching training and placebo computer. Dual-task performance benefits were assessed. Analyses compared percentage of change among groups in transfer dual-task conditions in three trial types: single-pure trials, single-mixed, and dualtask trials.

Results showed larger change in dual-mixed than in single-mixed trials, but only for the groups that completed the dual-task training (vs. placebo computer), with no substantial difference between aerobic and stretching exercise. Improvement in other task conditions was equivalent among all groups. Results of this study suggest that both dual-task computer training combined with aerobic or stretching can help improve the ability to maintain multiple stimulus-response alternatives. This specific skill is essential when it comes to concurrently performing multiple tasks. Moreover, these combined interventions also lead to functional improvement in balance and gait, but do not seem to have a synergetic effect, which could be partly explained by a reduced training volume due to the combination of multiple training.

These results further support the benefits of dual-task training on attentional control, and suggest equivalent effect of aerobic and stretching exercise on dual-task performance. 